\title{
Cardinal-indexed classifying spaces for families of subgroups of any topological group
}

\author{
Qayum Khan \\ Department of Mathematics \\ Indiana University \\ Bloomington IN 47405 USA
}

\begin{abstract}
For $G$ a topological group, existence theorems by Milnor (1956), Gelfand-Fuks (1968), and Segal (1975) of classifying spaces for principal $G$-bundles are generalized to $G$-spaces with torsion. Namely, any $G$-space approximately covered by tubes (a generalization of local trivialization) is the pullback of a universal space indexed by the orbit types of tubes and cardinality of the cover. For $G$ a Lie group, via a metric model we generalize the corresponding uniqueness theorem by Palais (1960) and Bredon (1972) for compact $G$. Namely, the $G$-homeomorphism types of proper $G$-spaces over a metric space correspond to stratified-homotopy classes of orbit classifying maps.

The former existence result is enabled by Segal's clever but esoteric use of non-Hausdorff spaces. The latter uniqueness result is enabled by our own development of equivariant ANR theory for noncompact Lie $G$. Applications include the existence part of classification for unstructured fiber bundles with locally compact Hausdorff fiber and with locally connected base or fiber, as well as for equivariant principal bundles which in certain cases via other models is due to Lashof-May (1986) and to Lück-Uribe (2014). From a categorical perspective, our general model $E_{\mathcal{F}}^{\kappa} G$ is a final object inspired by the formulation of the Baum-Connes conjecture (1994).
\end{abstract}

Keywords: classifying space, transformation group, stratified space, equivariant absolute neighborhood retract, noncompact Lie group

2020 MSC: 54H11, 55R15, 58A35, 54C55, 57S20

\section{Introduction}

Let $G$ be a topological group. Throughout this paper, we mostly consider right $G$-spaces $X$. Write $X / G:=\{x G \mid x \in X\}$ for its orbit space, where $x G:=\{x g \mid g \in G\}$ denotes an orbit. An isotropy group is the subgroup $G_{x}:=\{g \in G \mid x g=x\}$. For any subgroup $H$ of $G$, consider the right $H$-cosets $H g:=\{h g \mid h \in H\}$ and endow the right $G$-set $H \backslash G:=\{H g \mid g \in G\}$ with the quotient topology. The balanced product of a right $G$-space $X$ and a left $G$-space $Y$ is

$$
X \times{ }_{G} Y:=(X \times Y) /((x g, y) \sim(x, g y)) .
$$

Historically, several models of classifying spaces for principal $G$-bundles exist. Milnor (1956) introduced $\mathbb{E} G$ over base spaces $B$ that are paracompact Hausdorff, and Dold (1963)

\footnotetext{
*This paper is dedicated to Sergey Antonyan on the occasion of his 65th birthday

Email address: qkhan@indiana.edu (Qayum Khan)

Preprint submitted to Topology and its Applications
} 
proved a uniqueness theorem in which homotopy classes of maps $B \longrightarrow \mathbb{B} G:=\mathbb{E} G / G$ correspond to isomorphism clases of principal bundles over $B$. Gelfand-Fuks (1968) generalized this to all base spaces $B$ that are Tikhonov (that is, completely regular Hausdorff) via unnormalized joins, and Segal (1975) used non-Hausdorff cones to further observe a model $E G$ that works for $B$ being any topological space of arbitrary weight; however both are at the loss of uniqueness.

We extend Segal's model to a so-called $E_{\mathcal{F}} G$ to allow for fixed points with isotropy conjugate into a given set $\mathcal{F}$ of subgroups of $G$, where local triviality $U \times G$ of a principal bundle is replaced by the notion of an $H$-tube $S \times{ }_{H} G$, that is, the induction of a right $H$-space $S$ (called a slice) to a right $G$-space. Consequently, other models over more specialized base spaces admit a canonical $G$-map to this one, that is, it is more universal for $B$ any topological space. To avoid set-theoretic paradoxes, it is limited to $E_{\mathcal{F}}^{\kappa} G$ by the weight $w B \leqslant \kappa$ of $B$, which is the minimum cardinality for a base of the topology; for example, second-countable spaces have weight the first infinite cardinal $\boldsymbol{\aleph}_{0}$. If the covering of the $G$-space $X$ by tubes is approximate and $\mathcal{F}$ consists of closed subgroups, then our existence theorem (2.1) is that $X$ is the pullback of $E_{\mathcal{F}}^{\kappa} G$ along a map $X / G \longrightarrow E_{\mathcal{F}}^{\kappa} G / G$ whose explicit formula is canonically determined by the tube data with $\kappa=w(X / G)$.

Now assume $G$ is an arbitrary Lie group, such as a countable discrete group. Following Bredon's improvement (1972) of Palais' argument (1960) for compact Lie $G$, as well as employing and developing (3.28) modern advances in the equivariant theory of absolute neighborhood retracts (ANRs), we prove the following uniqueness theorem (4.7). Suppose that $X$ admits a $G$ invariant metric and that the action of $G$ is proper in the sense of Palais (1961), so an isovariant ( $H=G_{x}$ ) covering by tubes exists by Palais' slice theorem. Then the isomorphism classes of such $X$ over a given base space $B=X / G$ bijectively correspond to stratified-homotopy classes of maps $B \longrightarrow \mathbb{B}_{\mathcal{F}} G$, where $B$ has the induced orbit-type stratification and $\mathcal{F}$ is a set of compact subgroups of $G$ containing all the isotropy groups of $X$ without any conjugate representatives. Here $\mathbb{B}_{\mathcal{F}} G$ is the orbit space of the right $G$-space $\mathbb{E}_{\mathcal{F}} G$, which is our unnormalized join inspired by Gelfand-Fuks with coarse cones instead of fine ones. Palais-Bredon assume that $\mathcal{F}$ is finite so use normalized (Milnor) joins, as well as assume that $B$ is finite-dimensional; we remove these cardinal limitations, as well as no longer assume $G$ is compact whereby proper was automatic.

\section{Preliminaries}

M McCord introduced the following notion as non-Hausdorff cone [61, §8].

Definition 1.1 (McCord). Recall Sierpiński ${ }^{1}$ space $\mathbb{I}_{1}:=(\{0,1\},\{\varnothing,\{1\},\{0,1\}\})$. Let $(A, \mathcal{T})$ be a topological space. Write $A_{+}:=A \sqcup\{0\}$. The indiscrete cone is

$$
c(A, \mathcal{T}):=(A, \mathcal{T})_{+} \wedge \mathbb{I}_{1}=\left(A_{+}, \mathcal{T} \cup\left\{A_{+}\right\}\right)
$$

This let G Segal [72] cleverly simplify a construction of Gelfand-Fuks [39]. We generalize it here to non-free actions for which Segal's construction is $\mathcal{F}=\{1\}$.

Definition 1.2. Let $G$ be a topological group. Let $\mathcal{F}$ be any set of subgroups of $G$. Define $\mathcal{F} \backslash G:=\bigsqcup_{H \in \mathcal{F}} H \backslash G$ equipped with the coherent topology. Let $\kappa$ be a cardinal. Write $\kappa=\operatorname{card}(I)$ for a set $I$. Using the product and subspace topologies, define

$$
E_{\mathcal{F}}^{K} G:=(c(\mathcal{F} \backslash G))^{I}-\{0\}^{I}
$$

\footnotetext{
${ }^{1}$ Sierpiński $[73, \S 3, \S 9]$ noted $\mathbb{I}_{1}$ is the nondiscrete nonindiscrete Fréchet $\mathcal{V}$-space $[37, \mathrm{~V}]$ on two points. Open sets of a topological space correspond bijectively to continuous functions to $\mathbb{I}_{1}$.
} 
and the $\kappa$-indexed $\mathcal{F}$-classifying space $B_{\mathcal{F}}^{\kappa} G:=E_{\mathcal{F}}^{\kappa} G / G$ with quotient topology. Note the $G$ homeomorphism type of $E_{\mathcal{F}}^{\kappa} G$ does not depend on the representative $I$. For less cumbersome reading, we abbreviate $E^{\kappa} G:=E_{\{1\}}^{\kappa} G$ and $B^{\kappa} G:=B_{\{1\}}^{\kappa} G$.

We expand Biller's $[16,2.1]$ beyond Hausdorff $G$ and $X$ and compact $H \in \mathcal{F}$. Our expanded definition here also adds the notions of approximate and isovariant.

Definition 1.3. Let $G$ be a topological group. Let $X$ be a topological $G$-space. For any $x \in X$, its isotropy group is $G_{x}:=\{g \in G \mid x g=x\}$. Let $\mathcal{F}$ be any set of subgroups of $G$. An open $G$-subset $T$ of $X$ is an $\mathcal{F}$-tube if it is $G$-homeomorphic to $S \times{ }_{H} G$ for some $H \in \mathcal{F}$ and $H$-space $S$. We say that $X$ is covered by $\mathcal{F}$-tubes if $X=\bigcup_{i \in I} T_{i}$ for some $\mathcal{F}$-tubes $\left\{T_{i} \approx S_{i} \times_{H_{i}} G\right\}_{i \in I}$. More specifically, the cover is approximate if, for each point $x \in X$ and neighborhood $O$ of $G_{x}$ in $G$, there exist $i \in I$ and $g \in G$ with $x \in T_{i}$ and $G_{x} \leqslant g^{-1} H_{i} g \subset O$. In particular, the cover is isovariant if each $x \in X$ admits some $i \in I$ with $x \in T_{i}$ and $G_{x}$ conjugate to $H_{i}$.

(Isovariant) covering by $\mathcal{F}$-tubes implies tomDieck's “(strongly) locally $\mathcal{F}$-trivial” [27, p46].

Proposition 1.4. Any $E_{\mathcal{F}}^{\kappa} G$ can be covered by $\mathcal{F}$-tubes, in fact, by $\kappa \cdot \operatorname{card}(\mathcal{F})$-many. Also, the cover by $\mathcal{F}$-tubes is isovariant when restricted to the following $G$-subset:

$$
\mathscr{E}_{\mathcal{F}}^{K} G:=\left\{e \in E_{\mathcal{F}}^{\kappa} G \mid \exists i \in I: G_{e}=G_{e_{i}}\right\} .
$$

We shall call $\mathscr{B}_{\mathcal{F}}^{K} G:=\mathscr{E}_{\mathcal{F}}^{K} G / G$ the isovariant $\kappa$-indexed $\mathcal{F}$-classifying space. The $G$-space $\mathscr{E}_{\mathcal{F}}^{K} G$ is analogous to Palais' reduced join $[69,1.3 .6]\left[20\right.$, p108]. Note $\mathscr{E}_{\mathcal{F}}^{K} G$ is dense in $E_{\mathcal{F}}^{K} G$ if $\mathcal{F}$ is closed under conjugacy and $\kappa$-fold intersections.

Proof. Fix $i \in I$ and $H \in \mathcal{F}$. Define a $G$-space $T(i, H)$ and $H$-subspace $S(i, H)$ by

$$
\begin{aligned}
& T(i, H):=\left\{e \in E_{\mathcal{F}}^{\kappa} G \mid e_{i} \in H \backslash G\right\} \\
& S(i, H):=\left\{e \in E_{\mathcal{F}}^{\kappa} G \mid e_{i}=H \in H \backslash G\right\} .
\end{aligned}
$$

It remains to show that the following canonical bijective $G$-map is an open function:

$$
\mu: S(i, H) \times_{H} G \longrightarrow T(i, H) \quad ; \quad[e, g] \longmapsto e g=\left(e_{i} g\right)_{i \in I}
$$

Let $O$ be open in $G$. Let $j \neq i \in I$ and $K \in \mathcal{F}$. Let $U$ be open in $K \backslash G$. Consider

$$
B(j, U):=\left\{e \in S(i, H) \mid e_{j} \in U\right\} .
$$

For any $k \in I$, write $\pi_{k}:(c(\mathcal{F} \backslash G))^{I} \longrightarrow c(\mathcal{F} \backslash G)$ for the $k$-th projection $\left(e \mapsto e_{k}\right)$ and $V_{k}:=$ $\left(\pi_{k} \circ \mu\right)[B(j, U) \times O]$. Since $O$ is open $G$, note $V_{i}=\{H\} O=\{H\}\left(\bigcup_{h \in H} h O\right)$ is open in $H \backslash G$. Since $U$ is open in $K \backslash G$, note $V_{j}=U O=\bigcup_{g \in O} U g$ is open in $K \backslash G$ [27, I:3.1i]. Otherwise $V_{k}=c(\mathcal{F} \backslash G)$ is open in $c(\mathcal{F} \backslash G)$ for all $k \neq i, j$. Thus $\mu[B(j, U) \times O] \subseteq T(i, H)$ is open with respect to the product topology of $(c(\mathcal{F} \backslash G))^{I}$.

Observe that the subspace topology of $S(i, H)$ has subbase

$$
\mathcal{B}:=\{B(j, U) \mid j \in I-\{i\} \text { and } \exists K \in \mathcal{F}: U \text { is open in } K \backslash G\} .
$$

Let $B=B_{1} \cap \cdots \cap B_{n}$ be in the base generated by $\mathcal{B}$. Since $\mu$ is injective, note

$$
\mu[B \times O]=\mu\left[B_{1} \times O \cap \cdots \cap B_{n} \times O\right]=\mu\left[B_{1} \times O\right] \cap \cdots \cap \mu\left[B_{n} \times O\right]
$$


is open in $T(i, H)$. Thus $\mu\left[O^{\prime} \times O\right]$ is open in $T(i, H)$ for any open set $O^{\prime}$ in $S(i, H)$. Therefore $\mu[W]$ is open for any open set $W$ in product topology of $S(i, H) \times G$. As [-] $: S(i, H) \times$ $G \longrightarrow S(i, H) \times_{H} G$ is continuous, $\mu$ is open so a $G$-homeomorphism. Hence each $e \in E_{\mathcal{F}}^{\kappa} G=$ $(c(\mathcal{F} \backslash G))^{I}-\{0\}^{I}$ is a member of some $\mathcal{F}$-tube $T(i, H)$.

Finally, let $e \in \mathscr{E}_{\mathcal{F}}^{K} G$. Then $G_{e}=G_{e_{i}}$ for some $i \in I$. We may assume $e_{i} \neq 0$. Then $e_{i} \in H \backslash G$ for some $H \in \mathcal{F}$. So $e \in T(i, H) \cap \mathscr{E}_{\mathcal{F}}^{\kappa} G \approx\left(S(i, H) \cap \mathscr{E}_{\mathcal{F}}^{\kappa} G\right) \times_{H} G$.

\section{The classifying property: existence}

Theorem 2.1. Let $G$ be a topological group. Let $\mathcal{F}$ be any set of subgroups of $G$. Let $\kappa$ be a cardinal. Let $X$ be a G-space isovariantly covered by $\kappa$-many $\mathcal{F}$-tubes. Then $X$ is G-homeomorphic to the pullback $f^{*}\left(\mathscr{E}_{\mathcal{F}}^{\kappa} G\right)$ for some map $f: X / G \longrightarrow \mathscr{B}_{\mathcal{F}}^{\kappa} G$. The conclusion holds with $E_{\mathcal{F}}^{\kappa} G$ if the cover is only approximate and each $G_{x}$ closed.

Recall that each isotropy group $G_{x}$ is closed if $X$ is Hausdorff $\left(T_{2}\right)$ [27, I:3.5]. Any space is regular if any neighborhood of a point has a closed subneighborhood.

Proof. Let $i \in I$. There is a $G$-homeomorphism $\phi_{i}: T_{i} \longrightarrow S_{i} \times_{H_{i}} G$ with $H_{i} \in \mathcal{F}$. Write $q_{i}: S_{i} \times_{H_{i}} G \longrightarrow H_{i} \backslash G$ for the $G$-map $[s, g] \longmapsto H_{i} g$. Define a $G$-map

$$
F_{i}: X \longrightarrow c(\mathcal{F} \backslash G) \quad ; \quad x \longmapsto \begin{cases}\left(q_{i} \circ \phi_{i}\right)(x) & \text { if } x \in T_{i} \\ 0 & \text { if } x \notin T_{i} .\end{cases}
$$

The $G$-map $F: X \longrightarrow E_{\mathcal{F}}^{\kappa} G$ whose $i$-th coordinate is $F_{i}$ induces $f: X / G \longrightarrow B_{\mathcal{F}}^{\kappa} G$. It remains to prove the following canonical surjective $G$-map is an open injection:

$$
\Psi: X \longrightarrow f^{*}\left(E_{\mathcal{F}}^{\kappa} G\right) \quad ; \quad x \longmapsto(x G, F(x)) .
$$

Fix $i \in I$. Let $O$ be open in $G$, and let $U$ be open in $S_{i}$. Consider the open set

$$
V:=\phi_{i}^{-1}[U \times G] G \times\left\{e \in E_{\mathcal{F}}^{\kappa} \mid e_{i} \subset H_{i} O\right\}
$$

in $X / G \times E_{\mathcal{F}}^{\kappa} G$, with the quotient map $X \longrightarrow X / G ; x \longmapsto x G$ open [27, I:3.1iv]. Note $\Psi\left(\phi_{i}^{-1}[U \times\right.$ $\left.\left.H_{i} O\right]\right)=V \cap f^{*}\left(E_{\mathcal{F}}^{\kappa} G\right)$. Thus $\Psi \mid T_{i}$, hence $\Psi$, is an open function.

It remains to show that $\Psi$ is injective. Suppose $\Psi(x)=\Psi(y)$ for some $x, y \in X$. Then $x G=y G$, that is, $y=x a$ for some $a \in G$. First, assume the cover is isovariant. There exist $i \in I$ and $g \in G$ such that $x \in T_{i}$ and $G_{x}=g^{-1} H_{i} g$. Note $F_{i}(x)=H_{i} n g$ for some $n \in N_{G}\left(H_{i}\right)$. Then $H_{i} n g a=F_{i}(x) a=F_{i}(y)=F_{i}(x)=H_{i} n g$. So $a \in G_{x}$. Hence $y=x$. Alternatively, assume the cover is approximate and $G_{x}$ is closed in $G$. Kolmogorov proved topological groups $G$ are regular in the above sense [50, §1]. Assume $a \notin G_{x}$. Then $a \notin O$ for some neighborhood $O$ of $G_{x}$ in $G$. There are $i \in I$ and $g \in G$ with $x \in T_{i}$ and $G_{x} \leqslant g^{-1} H_{i} g \subset O$. Again note $F_{i}(x)=H_{i} n g$ and then $\mathrm{gag}^{-1} \in H_{i}$. So now $a \in O$, a contradiction. Hence $a \in G_{x}$. Therefore $y=x$.

Remark 2.2. Below are earlier classifying spaces that isovariantly map to ours. Our models $E_{\mathcal{F}}^{\kappa} G$ are $T_{0}$ but not $T_{1}$, if $G$ is $^{2} T_{0}$ and each $H \in \mathcal{F}$ is a closed set in $G$. A reason to regard higher cardinals $\kappa$ is $G=\left(S^{1}\right)^{I}$, with the product topology, for any infinite set $I$. These infinite-dimensional toral groups are connected compact [22, p830] abelian Hausdorff groups and archetypes beyond Lie groups $[43,8.15]$. The continuum $c:=2^{\aleph_{0}}$ can be $\boldsymbol{\aleph}_{\alpha}$ for ordinals $\alpha>0$ in ZFC set theory.

${ }^{2}$ Kolmogorov $\left(T_{0}\right)$ topological groups $G$ are Tikhonov $\left(T_{3.5}\right.$, completely regular Hausdorff) [71, Tеорема 10]. 


\subsection{Free actions}

The following was my motivation and stated by G Segal in russian [72].

Corollary 2.3 (Segal). Let $G$ be any topological group. Let $\kappa$ be any cardinal. Let $X$ be a principal G-bundle covered by $\kappa$-many local trivializations. Then $X$ is $G$-homeomorphic to the pullback bundle $f^{*}\left(E^{\kappa} G\right)$ for some map $f: X / G \longrightarrow B^{\kappa} G$.

This simplified Gelfand-Fuks' model [39] ${ }^{3}$ where the orbit space $X / G$ is assumed Tikhonov $\left(T_{3.5}\right)$ : a $T_{0}$ space $Y$ is Tikhonov if points and closed sets are separated by maps $Y \longrightarrow[0,1]$. An upper bound on $\kappa$ is the weight of $X / G$, the minimum cardinality for a base. For example, if $X / G$ is second-countable, then $\kappa$ can be taken as the first infinite cardinal $\boldsymbol{\aleph}_{0}$.

Corollary 2.4 (Gelfand-Fuks). Let $G$ be a topological group. Let B be a Tikhonov space, with weight denoted $w B$. Let $X$ be a principal $G$-bundle over $B$. Then $X$ is $G$-homeomorphic to the pullback bundle $f^{*}\left(E^{w B} G\right)$ for some map $f: B \longrightarrow B^{w B} G$.

This served to generalize Dold's pullback [30] of Milnor's construction [63]. A fast formula for $f$ is given by tomDieck [25, II] and Husemöller [47, 4:12.2], who applied Milnor's countable partition of unity trick [64, p25-26] [66, 5.9].

Corollary 2.5 (Milnor-Dold). Let $G$ be a topological group. Let B be a paracompact Hausdorff space. Let $X$ be a principal $G$-bundle over $B \approx X / G$. Then $X$ is $G$-homeomorphic to the pullback bundle $f^{*}\left(E^{\aleph_{0}} G\right)$ for some map $f: B \longrightarrow B^{\aleph_{0}} G$.

In turn, this bests Steenrod [75, 19.6, 19.3]: $O_{n} \backslash O_{n+k}$ models $E^{n} G$ for an embedding $G \leqslant O_{k}$.

Corollary 2.6 (Steenrod). Let $G$ be a compact Lie group. Let $B$ be a finite simplicial complex, say of dimension $n$. Let $X$ be a principal $G$-bundle over $B$. Then $X$ is $G$-homeomorphic to the pullback bundle $f^{*}\left(E^{n} G\right)$ for some map $f: B \longrightarrow B^{n} G$.

The same conclusion holds for $G$ Lie and $B$ paracompact of $\operatorname{dim} \leqslant n[49,5.10]$.

Remark 2.7. We illustrate how Segal's allowance of non-Hausdorff base spaces (2.3) is useful in geometric combinatorics beyond Milnor-Dold's model (2.5). Let $G$ be a discrete finite group. Recall that a $G-C W$ complex is regular if the attaching map of each cell is a homeomorphism. Consider the problem of enumerating, possibly with repetition of isomorphism classes, all free regular $G$-CW complexes $E$ with orbit space a given connected finite regular CW complex $B$.

By functoriality in Björner's correspondence [17, 3.1], regular $G$-CW complexes (each admits a canonical simplicial subdivision [58, III:2.1, III:1.7]) correspond (via the face poset consisting of the closed cells under inclusion) to so-called CW posets [17, 2.1] with $G$-action; here "poset" abbreviates "partially ordered set." Given any CW complex $K$, write $\Delta(K)$ for its face poset and consider the Aleksandrov-discrete space $\|\Delta(K)\|$ : the $T_{0}$ (which is $T_{1}$ iff $\Delta(K)$ has no comparable elements iff $\operatorname{dim}(K)=0$ ) topological space on the underlying set of $\Delta(K)$ with the open sets being the upper sets $U$ (that is, $y \supseteq x \in U$ implies $y \in U$ ) [3]. Then, by Segal's model (2.3), enumeration of all free regular $G$-CW complexes $E$ with $E / G=B$, possibly repeating isomorphism classes, is all of the maps $A:=\|\Delta(B)\| \longrightarrow B^{w A} G$ between these finite $T_{0}$ spaces.

\footnotetext{
${ }^{3}$ Also [39] had only $X / G$ be Hausdorff if $X$ were "locally T-trivial" like [33, Définition(a)].
} 
On the other hand, if one uses the Milnor-Dold model (2.5) in conjunction with obstruction theory, all connected free (hence CW) $G$-spaces $X$ with $X / G=B$ would correspond to the classification of regular $G$-fold coverings: the conjugacy classes of normal subgroups of $\pi_{1}(B)$ with quotient $G$. In principle, for small $G$ and finitely presented $\pi_{1}(B)$, the Dietze-Schaps multistep algorithm [28] applies to this classical enumeration; however one inputs the order of $G$ and then eliminates the spurious quotient groups of the same order. Alternatively, our new perspective in terms of conversion to finite $T_{0}$ spaces directly provides a finite search-space implementable on a computer; a second pass eliminates redundant representatives within isomorphism classes.

For nonfree actions of finite groups $G$ with orbit space a finite regular CW complex $B$, using Mostow's slice theorem for these $T_{3.5} G$-spaces to obtain $\mathcal{F}$-tubes [69, 1.7.19], in principle our method (2.1) of finite $T_{0}$ spaces works. On the other hand, the classical approach of PalaisBredon $(2.14,4.7)$ involves (stratified) obstruction theory - a multistep cohomological process.

\subsection{Unstructured fiber bundles}

Balanced products $X \times{ }_{G} F$ allow analogies of the above results for fiber bundles with fiber $F$ and structure group $G[75,2.3]$. However, applications do not effortlessly and formally occur to the more primitive notion of a fiber bundle with fiber $F$ and no given structure group [75, 1.1].

Nonetheless, for any base and certain fibers, we can combine [75, 5.4-5.5] with [12, Theorem 4] to associate a principal bundle to unstructured fiber bundles.

Theorem 2.8 (Steenrod-Arens). Let $F$ be a Hausdorff space that either is compact or is both locally connected and locally compact. Endow $\operatorname{Homeo}(F)$ with compact-open topology $G$ [36]. Any $F$-fiber bundle over any topological space $B$ is isomorphic to the balanced product $X \times_{G} F$ for some principal G-bundle X over $B$.

Corollary 2.9. Let $F$ be compact $T_{2}$ space or locally connected locally compact $T_{2}$. Endow Homeo $(F)$ with compact-open topology $G$. Let $B$ be a topological space; let $\kappa$ be a cardinal. Any $F$-fiber bundle $p: E \longrightarrow B$ covered by $\kappa$-many local trivializations $\left\{U_{i} \times F\right\}_{i \in I}$ is isomorphic to pullback $f^{*}\left(E^{\kappa} G \times{ }_{G} F\right)$ for a map $f: B \longrightarrow B^{\kappa} G$.

Proof. This is immediate from Theorem 2.8 and Corollary 2.3.

Remark 2.10 (Cianci-Ottina). For $B$ any Aleksandrov space [3] and the above sort of fiber $F$, Corollary 2.9 overlaps with existence of a Grothendieck-type classifying space for unstructured $F$-fiber bundles over $B$ found recently in $[23,4.3]$.

The following result is well-known nowadays but seems to be undocumented.

Corollary 2.11 (Holm). Endow Homeo $\left(\mathbb{R}^{n}, 0\right)$ with compact-open topology $\mathrm{TOP}_{n}$. Let $B$ be a paracompact Hausdorff space. Any $\mathbb{R}^{n}$-microbundle $p: E \longrightarrow B$ [65] is isomorphic to pullback $f^{*}\left(E^{\aleph_{0}} \mathrm{TOP}_{n} \times_{\mathrm{TOP}_{n}} \mathbb{R}^{n}\right)$ for a map $f: B \longrightarrow B^{\aleph_{0}} \mathrm{TOP}_{n}$.

Proof. Holm shows $\mathbb{R}^{n}$-microbundles over $B$ are $\left(\mathbb{R}^{n}, 0\right)$-fiber bundles [44, 3.3]. As $\mathbb{R}^{n}$ is locally connected locally compact, use Theorem 2.8 and Corollary 2.5.

Analyzing his own main proof $(Y=[0,1])$, Crowell noticed this fact $[24, \S 4]$.

Theorem 2.12 (Crowell). Let $X$ be any locally compact Hausdorff space. Endow Homeo(X) with Arens' g-topology [12]. Let $Y$ be any locally connected space. Any continuous function $h: X \times Y \longrightarrow X$ with each $h_{y}: X \longrightarrow X ; x \longmapsto h(x, y)$ a homeomorphism has its adjoint $h^{*}: Y \longrightarrow \operatorname{Homeo}(X) ; y \longmapsto h_{y}$ being continuous. 
Now, we allow the fibers of Corollary 2.9 to include non-locally connected examples, such as the $p$-adic rationals $F=\mathbb{Q}_{p}$, by transferring the condition to the base.

Corollary 2.13. Let $F$ be any locally compact Hausdorff space. Endow $\operatorname{Homeo}(F)$ with Arens' g-topology $G$. Let $B$ be any locally connected topological space; let $\kappa$ be a cardinal. Any $F$-fiber bundle $p: E \longrightarrow B$ covered by $\kappa$-many local trivializations $\left\{U_{i} \times F\right\}_{i \in I}$ is isomorphic to the pullback $f^{*}\left(E^{\kappa} G \times{ }_{G} F\right)$ for a map $f: B \longrightarrow B^{\kappa} G$.

Proof. By [12, Theorem 3], $G$ is a topological group with continuous evaluation function $G \times$ $F \longrightarrow F ;(g, f) \longmapsto g(f)$, and it is the coarsest for which these hold. For any transition $\left(U_{i} \cap U_{j}\right) \times F \longrightarrow\left(U_{i} \cap U_{j}\right) \times F \stackrel{\mathrm{pr}_{F}}{\longrightarrow} F$ of local trivializations, since $U_{i} \cap U_{j} \subset B$ is locally connected, by Theorem 2.12, its adjoint $U_{i} \cap U_{j} \longrightarrow G$ is continuous. So the $F$-fiber bundle $p: E \longrightarrow B$ has structure group $G[75,2.3]$ and is isomorphic to $X \times_{G} F$ for an associated $G$-principal bundle $X \longrightarrow B[75,8.1]$. Then $X$ is $G$-homeomorphic over $B$ to a pullback of $E^{\kappa} G$, by Corollary 2.3 .

Again, notice that an upper bound on the cardinal $\kappa$ is the weight $w B$ of the base space $B$.

\subsection{Nonfree actions}

Bredon [20, II:9.7i] reworked Palais [69, 2.6.2], who had $X$ separable and locally compact. In the conclusion [70, 4.5], Palais asserts that his classification also holds for any Lie group $G$ if the action is Palais-proper; this assertion is enacted and extended further in our Theorem 4.7.

Corollary 2.14 (Palais-Bredon). Let $G$ be a compact Lie group. Let $\mathcal{F}$ be a finite set of subgroups of $G$ with no conjugate elements. Let $X$ be a metrizable $G$-space with all orbit types represented in $\mathcal{F}$. Then $X$ is $G$-homeomorphic to the pullback $f^{*}\left(\mathscr{E}_{\mathcal{F}}^{\aleph_{0}} G\right)$ for some map $f: X / G \longrightarrow \mathscr{B}_{\mathcal{F}}^{\aleph_{0}} G$.

Recall, when $G$ is a compact Lie group, the Peter-Weyl theorem implies that there are only countably many conjugacy classes of compact subgroups [69, 1.7.27]. Next, Ageev had a similar construction to ours in the realm of metric spaces [2, 3.2].

Corollary 2.15 (Ageev). Let $G$ be a compact Lie group. Write cpt for the set of compact subgroups of $G$. Let $X$ be a metrizable $G$-space (hence $X / G$ is metrizable). Then $X$ is $G$ homeomorphic to pullback $f^{*}\left(E_{\mathrm{cpt}}^{\aleph_{0}} G\right)$ for a map $f: X / G \longrightarrow B_{\mathrm{cpt}}^{\aleph_{0}} G$.

Now we generalize this further, from having $X / G$ be metrizable to only Tikhonov.

Corollary 2.16. Let $G$ be any Lie group. Write cpt for the set of compact subgroups of G. Let $X$ be a Tikhonov space, equipped with a Palais-proper G-action. By Palais' slice theorem [70], $X$ is isovariantly covered by cpt-tubes, say $\kappa$-many. Then $X$ is G-homeomorphic to the pullback $f^{*}\left(E_{\mathrm{cpt}}^{\kappa} G\right)$ for some map $f: X / G \longrightarrow B_{\mathrm{cpt}}^{\kappa} G$.

In the next definition, the trivial group is in cpt but not in $\operatorname{lrg}$ for the $p$-adic integers $G=\mathbb{Z}_{p}$.

Definition 2.17 (Antonyan). Let $G$ be a locally compact Hausdorff group. A subgroup $H$ of $G$ is large if the homogeneous space $G / H$ is a topological manifold. Write $\operatorname{lrg} \subseteq \operatorname{cpt}$ (equality if $G$ Lie) for the subset of large compact subgroups of $G$.

The approximate-slice theorem of Abels-Biller-Antonyan [10, 3.6] is used instead of Palais' slice theorem to prove the following generalization of Corollary 2.16. Our conclusion follows from theirs: their $G$-map $F$ is an embedding [6, 4.4(1)]. We conclude the same; our $F$ also separates points from closed sets $[35,2.3 .20]$. 
Corollary 2.18 (Antonyan-Antonyan-Valera-Velasco). Let $G$ be a locally compact Hausdorff group. Let $X$ be a Tikhonov space with a Palais-proper G-action. Then $X$ is G-homeomorphic to the pullback $f^{*}\left(E_{\operatorname{lrg}}^{w X} G\right)$ for some map $f: X / G \longrightarrow B_{\operatorname{lrg}}^{w X} G$.

Proof. By Theorem 2.1 it suffices to approximately cover $X$ by $w X$-many lrg-tubes.

Let $x \in X$; let $O$ be an open neighborhood of $G_{x}$ in $G$. Since the $G$-action on $X$ is Bourbakiproper [16, 1.4, 1.6c], the $G$-map $G \longrightarrow x G ; g \longmapsto x g$ is open [27, I:3.19iii]. Then $x O=U \cap x G$ for an open set $U$ in $X$. By the approximate-slice theorem [10,3.6], there exists a large compact subgroup $H \geqslant G_{x}$ of $G$ with $x H \subset U$. Then $x H \subset x O$. Hence $H \subset O$. Moreover, that theorem gives an open $G$-neighborhood $T(x, O)$ of $x$ in $X$ that is $G$-homeomorphic to $S \times{ }_{H} G$ for some $H$-space $S[1,3.5]$. Finally, this approximate cover $\{T(x, O)\}$ of $X$ by lrg-tubes, by the Axiom of Choice, has a subcover ${ }^{4}$ of cardinality $\leqslant w X[35,1.1 .14]$.

\subsection{Generalized equivariant principal bundles}

Theorem 2.19. Let $\Gamma$ be a topological group; let $\Pi$ be any normal subgroup of $\Gamma$. Let $\mathcal{F}$ be a set of subgroups of $\Gamma$ such that $H \cap \Pi=1$ for each $H \in \mathcal{F}$. Let $\kappa$ be a cardinal. Let $X$ be a $\Gamma$-space isovariantly covered by $\kappa$-many $\mathcal{F}$-tubes. Then $X$ is $\Gamma$-homeomorphic to the pullback $c^{*}\left(\mathscr{E}_{\mathcal{F}}^{K} \Gamma\right)$ for some $\Gamma / \Pi$-map $c: X / \Pi \longrightarrow \mathscr{E}_{\mathcal{F}}^{k} \Gamma / \Pi$.

Observe $X \longrightarrow X / \Pi$ is a principal $\Pi$-bundle, as the restriction of $\mathcal{F}$ to $\Pi$ is $\{1\}$.

Proof. Define $c$ using the $F$ and $f$ for $\Gamma$ of Proof 2.1 in the commutative diagram

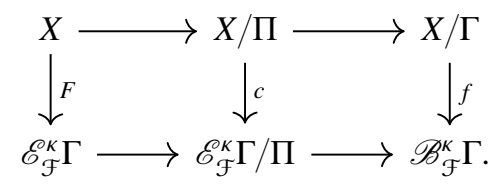

Then $X$ is $\Gamma$-homeomorphic to the pullback $f^{*}\left(\mathscr{E}_{\mathcal{F}}^{\kappa} \Gamma\right)$, by Theorem 2.1. Consider the set $\mathcal{F}_{\Pi}:=$ $\left\{H_{\Pi}:=H \Pi / \Pi \mid H \in \mathcal{F}\right\}$ of subgroups of the topological group $G:=\Gamma / \Pi$.

Note $G_{x \Pi}=\left(\Gamma_{x}\right)_{\Pi}$. For each $H \in \mathcal{F}$, the quotient map $H \longrightarrow H_{\Pi}$ is an isomorphism, since $H \cap \Pi=1$. Then any $H$-space $S$ is an $H_{\Pi}$-space. So $\left(S \times_{H} \Gamma\right) / \Pi \cong S \times_{H_{\Pi}} G$ as $G$-spaces. Thus, by Proof 1.4, the $G$-space $\mathscr{E}_{\mathcal{F}}^{K} \Gamma / \Pi$ is isovariantly covered by $\kappa \cdot \operatorname{card}(\mathcal{F})$-many $\mathcal{F}$-tubes. Theorem 2.1 gives the commutative diagram

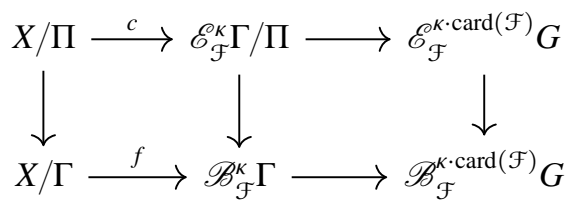

where the right square and the rectangle are pullbacks. By the so-called pasting law [60, III:4.8b], the left square of (2.2) is a pullback. Again, since the right square and the rectangle of (2.1) are pullbacks, the left square of (2.1) is a pullback.

${ }^{4}$ Note Lindelöf's lemma is $w X=\aleph_{0}$ : any open cover has a countable subcover [55, II: $\mathbb{R}^{n}$ ]. 
May-Elmendorf's model [34, p278] led to this existence part of [52, Theorem 9]. The pullback property [51, p269] is established in [53, Theorem 7].

Corollary 2.20 (Lashof-May-Rothenberg). Let $\Pi$ be a closed normal subgroup of a compact Lie group $\Gamma$. Write $\mathcal{F}(\Pi ; \Gamma)$ for the set of closed subgroups $H$ of $\Gamma$ with $H \cap \Pi=1$. Let $X \longrightarrow X / \Pi$ be a numerable $(\Pi ; \Gamma)$-bundle [52] with X Tikhonov. Suppose that $X$ has the $\Gamma$-homotopy type of a $\Gamma$-CW complex. ${ }^{5}$ Then the $\Gamma$-space $X$ is $\Gamma$-homeomorphic to $c^{*}\left(\mathscr{E}_{\mathcal{F}(\Pi ; \Gamma)}^{\mathrm{N}_{0}} \Gamma\right)$ for some $\Gamma / \Pi$-map $c: X / \Pi \longrightarrow \mathscr{E}_{\mathcal{F}(\Pi ; \Gamma)}^{\aleph_{0}} \Gamma / \Pi$.

Proof. An isovariant cover exists by Palais [70]; $\kappa=\boldsymbol{\aleph}_{0}$ by tomDieck [25].

A noncompact result exists for twisted equivariant principal bundles [57, 11.4].

Corollary 2.21 (Lück-Uribe). Let $\Pi, G$ be compactly generated Hausdorff groups. Let $\tau$ : $G \longrightarrow \operatorname{Aut}(\Pi)$ be a homomorphism with adjoint $G \times \Pi \longrightarrow \Pi$ continuous. Write $\Gamma:=k\left(\Pi \rtimes_{\tau} G\right)$ [18, III:2.28] [76]. Let $X$ be a $\Gamma$-CW complex with $X \longrightarrow X / \Pi a(G, \tau)$-equivariant principal $\Pi$-bundle. Suppose $\mathcal{R}$ is a family of local representations for $(G, \tau, \Pi)[57,3.3]$ that satisfies the $(H)$-condition [57, 6.1]. Write $\mathcal{F}(\mathcal{R})$ for its associated family of closed subgroups of $\Gamma$ [57, 3.5]. Then $X$ is $\Gamma$-homeomorphic to the pullback $c^{*}\left(\mathscr{E}_{\mathcal{F}(\mathcal{R})}^{\aleph_{0}} \Gamma\right)$ for a G-map $c: X / \Pi \longrightarrow \mathscr{E}_{\mathcal{F}(\mathcal{R})}^{\aleph_{0}} \Gamma / \Pi$.

Remark 2.22 (Guillou-May-Merling). Let $\Pi$ be either a discrete group or a compact Lie group. Let $G$ be a discrete group. Let $\tau: G \longrightarrow \operatorname{Aut}(\Pi)$ be any homomorphism. Write $\Gamma:=\Pi \rtimes_{\tau} G$. Corollary 2.21 applies with $\mathcal{F}(\mathcal{R})=\mathcal{F}(\Pi ; \Gamma)$. The model in $[41,0.4]$ is more rigid, as it descends from a categorical framework.

\section{The classifying property: uniqueness, I}

\subsection{Topological groups $G$ and arbitrary $G$-spaces}

As a prelude to the next subsection, we discuss coarse cones and coarse joins (survey in [38]).

Definition 3.1. Let $(A, \mathcal{T})$ be a topological space. Consider the half-smash set

$$
A_{+} \wedge[0,1]:=A \times[0,1] /\left(\forall a, a^{\prime} \in A:(a, 0) \sim\left(a^{\prime}, 0\right)\right) .
$$

The coarse cone $C(A, \mathcal{T})$ is this set equipped with the coarsest topology for which the functions $A_{+} \wedge[0,1] \longrightarrow[0,1] ;[a, t] \longmapsto t$ and $A \times(0,1] \longrightarrow A ;[a, t] \longmapsto a$ are continuous. The fine cone $\mathscr{C}(A, \mathcal{T})$ is that set equipped with the finest topology for which the function $A \times[0,1] \longrightarrow$ $A_{+} \wedge[0,1] ;(a, t) \longmapsto[a, t]$ is continuous.

Remark 3.2. For any space $X$, the identity function $\mathscr{C} X \longrightarrow C X$ is continuous. It is a homeomorphism if $X$ is compact, by the tube lemma. It is not so for $X=\mathbb{R}$, since $\{[x, t] \in$ $\left.\mathbb{R}_{+} \wedge[0,1] \mid t<1 /\left(1+x^{2}\right)\right\}$ is a neighborhood of the cone point in the fine topology but not the coarse one. If $d$ is a metric on $X$ then $C X$ has metric

$$
C d([x, s],[y, t]):=|s-t|+\min \{s, t\} \cdot d(x, y) .
$$

Note a function $f=\left(f_{0}, f_{1}\right): A \longrightarrow C X$ is continuous if and only if its coordinates $f_{1}: A \longrightarrow$ $[0,1]$ and $f_{0}: f_{1}^{-1}(0,1] \longrightarrow X$ are continuous. A function $\mathscr{C} X \longrightarrow Z$ is continuous if and only if the composition with $X \times[0,1] \longrightarrow \mathscr{C} X$ is continuous.

\footnotetext{
${ }^{5} \mathrm{~A}(\Pi ; \Gamma)$-bundle $X \longrightarrow X / \Pi$ is numerable and $X \in T_{3.5}$ if $X$ is a $\Gamma$-CW complex [52, 4,5].
} 
Remark 3.3. Historically, these two notions of cone were implicit in the topological study of simplicial complexes $K$. If $K$ is given the CW topology [82, p316], then $\mathscr{C} K$ is canonically a CW complex. If $K$ is given the euclidean-metric topology [54, I.1:4.12], then $C K$ is induced by the euclidean metric ${ }^{6}$. Recall the $\mathrm{CW}$ topology on $K$ is finer than the metric one and is it if and only if $K$ is locally finite.

If $X$ is a $G$-space, then $C X$ and $\mathscr{C} X$ have $G$-actions defined by $[x, t] \cdot g:=[x g, t]$.

Example 3.4 (Gelfand-Fuks). Their unrestricted join [39] of any $G$-spaces is

$$
\underset{i \in I}{*} X_{i}:=\left(\prod_{i \in I} \mathscr{C} X_{i}\right)-\{0\}^{I}
$$

Correspondingly, we reformulate Milnor's definition [63] in terms of cones.

Definition 3.5 (Milnor). The coarse join of any set of topological spaces is

$$
\bigcirc_{i \in I} X_{i}:=\left\{[x, t] \in \prod_{i \in I} C X_{i} \mid \exists \text { finite } J \subseteq I: \sum_{j \in J} t_{j}=1 \text { and } \forall i \in I-J: t_{i}=0\right\}
$$

endowed with the subspace topology induced from Tikhonov's product topology. Write $\mathbb{E} Z:=$ $Z^{\circ \aleph_{0}}$ for any space $Z$, with the diagonal $G$-action if $Z$ has a $G$-action.

As just above, we update for arbitrary cardinality, in [69, 1.3.6] [20, p108].

Definition 3.6 (Palais). The isovariant join of a set of topological $G$-spaces is

$$
\underset{i \in I}{*} X_{i}:=\left\{[x, t] \in \bigcirc_{i \in I} X_{i} \mid \exists i \in I: G_{[x, t]}=G_{x_{i}}\right\} .
$$

However, if $I$ is infinite, this 'hemorrhages' in neighborhoods of $A \cup X_{(H)}$ in $X$ in [20, Proof II:9.5], obstructing renormalization on $X-A$ to finite support. This naïveté is untenable for Proof 3.28; we introduce our own coarse join in the Gelfand-Fuks style, which for finite $I$ is $G$-homeomorphic to Palais' join via normalization.

Definition 3.7. The unrestricted isovariant join of topological $G$-spaces is

$$
\underset{i \in I}{*} X_{i}:=\left\{[x, t] \in \prod_{i \in I} C X_{i}-\{0\}^{I} \mid \exists i \in I: G_{[x, t]}=G_{x_{i}}\right\} .
$$

We remind the reader of the following notion of a proper action $[70,1.2 .2]$. Note that the Palais-proper condition is automatic if $G$ is an arbitrary compact group.

Definition 3.8 (Palais). A topological $G$-space $X$ is Palais if every $x \in X$ has a neighborhood $U$ in $X$ satisfying: each $y \in X$ has a neighborhood $V$ in $X$ so that the $\operatorname{transporter}\langle U, V\rangle_{G}:=\{g \in$ $G \mid U g \cap V \neq \varnothing\}$ is precompact (that is, has compact closure). Observe that if $G$ is discrete, then $\langle U, U\rangle_{G}$ being precompact (hence finite) means that the action is properly discontinuous.

\footnotetext{
${ }^{6}$ Given the vertex set $S$ of the abstract simplicial complex [4, §IV.1:1] underlying $K$, there is a geometric realization in terms of basis vectors in the coproduct $\mathbb{R}^{\oplus S}$ equipped with the 2-norm.
} 
In the second half of this subsection, we quickly construct a filtered homotopy.

Definition 3.9. The following topological space we shall call bi-Sierpiński space:

$$
\mathbb{I}_{2}:=(\{-1,0,+1\},\{\varnothing,\{0\},\{-1,0\},\{0,+1\},\{-1,0,+1\}\}) .
$$

It is the particular-point topology on three elements where $\mathbb{I}_{1}$ is for two [74, II:8]. The inclusion $\mathbb{I}_{1} \longrightarrow \mathbb{I}_{2} ; 0,1 \longmapsto 1,0$ has left-inverse $\mathbb{I}_{2} \longrightarrow \mathbb{I}_{1} ;-1,0,1 \longmapsto 1,1,0$. Earlier, $\mathbb{I}_{2}$ occurs as the upper topology on the poset of the 1-simplex [4, I:1.4]. Notice the continuous surjection $\Delta:[-1,1] \longrightarrow \mathbb{I}_{2} ; \pm 1 \longmapsto \pm 1,-1<t<1 \longmapsto 0$.

Lemma 3.10. Let $G$ be a topological group. Let $\mathcal{F}$ be any set of subgroups of $G$. Suppose $\left\{T_{i} \approx G \times_{H_{i}} S_{i}\right\}_{i \in I}$ and $\left\{T_{j} \approx G \times_{H_{j}} S_{j}\right\}_{j \in J}$ each $\mathcal{F}$-isovariantly cover a $G$-space $X$ for sets $I$ and $J$. There is a G-map $\Phi: X \times \mathbb{I}_{2} \longrightarrow \mathscr{E}_{\mathcal{F}} \sqcup J G$ that restricts to the classifying $G$-maps $F_{-}: X \times\{-1\} \longrightarrow \mathscr{E}_{\mathcal{F}}^{I} G$ and $F_{+}: X \times\{+1\} \longrightarrow \mathscr{E}_{\mathcal{F}}^{J} G$. The same holds for approximate coverings by $\mathcal{F}$-tubes where $E_{\mathcal{F}}^{*} G(1.2)$ replace $\mathscr{E}_{\mathcal{F}}^{*} G$.

So the classifying maps $f_{ \pm}: X / G \longrightarrow \mathscr{B}_{\mathcal{F}}^{I \sqcup J} G$ are homotopic via $(\mathrm{id} \times \Delta) \circ \Phi / G$. The proof works more generally for $G$-maps $F_{ \pm}$not necessarily induced from tubes.

Proof. Define $\Phi \mid: X \times\{0\} \longrightarrow \mathscr{E}_{\mathcal{F}}{ }^{I} J$ to be the classifying $G$-map of Theorem 2.1 for the combined isovariant cover $\left\{T_{i}\right\}_{i \in I} \sqcup\left\{T_{j}\right\}_{j \in J}$ of the $G$-space $X$ by $\mathcal{F}$-tubes. Define $\Phi \mid X \times\{-1\}$ to be the classifying $G$-map $F_{-}$for the isovariant cover $\left\{T_{i}\right\}_{i \in I}$; define $\Phi \mid X \times\{+1\}$ to be the classifying $G$-map $F_{+}$for the isovariant cover $\left\{T_{j}\right\}_{j \in J}$. Here we use the $G$-embedding $\mathscr{E}_{\mathcal{F}} G \sqcup$ $\mathscr{E}_{\mathcal{F}}^{J} G \longrightarrow \mathscr{E}_{\mathcal{F}}^{I \sqcup J} G$ given by extension by zero.

Let $O$ be open in $G$. Let $i \in I$. In the product topology, consider the open set

$$
B(O, i):=\left\{e \in \mathscr{E}_{\mathcal{F}}^{I \sqcup J} G \mid e_{i} \in O H_{i} / H_{i}\right\}
$$

There is equipped a $G$-homeomorphism $\phi_{i}: T_{i} \longrightarrow G \times_{H_{i}} S_{i}$. Note the preimage

$$
\Phi^{-1}(B(O, i))=\phi_{i}^{-1}\left(O H_{i} \times_{H_{i}} S_{i}\right) \times\{-1,0\}
$$

is open in $X \times \mathbb{I}_{2}$. Similarly one defines $B(O, j)$ for any $j \in J$ and verifies a similar equality. Observe that $\{B(O, k) \mid O$ open in $G$ and $k \in I \sqcup J\}$ is a subbase for the topology of $\mathscr{E}_{\mathcal{F}} I \sqcup J$. Therefore the $G$-function $\Phi$ is continuous.

Theorem 3.11. Let $G$ be a topological group. Let $\mathcal{F}$ be any set of subgroups of $G$. Let $\kappa$ be an infinite cardinal. Then $E_{\mathcal{F}}^{\kappa} G$ is a final object in the category of all topological G-spaces covered by $\kappa$-many $\mathcal{F}$-tubes and $G$-homotopy classes of $G$-maps.

For paracompact Hausdorff orbit space, one assumes $\kappa=\boldsymbol{\aleph}_{0}$ by Proposition 4.4.

Proof. Existence is in Proof 2.1, without "approximate" for the extra "pullback." Uniqueness up to $G$-homotopy is Lemma 3.10 via $(\mathrm{id} \times \Delta) \circ \Phi$ and $\kappa+\kappa=\kappa$.

Here is an important case [27, I:6.6], upon which the Baum-Connes conjecture is formulated. Asserted in [15, Proof A:1], one must replace Husemöller's case of $\mathcal{F}=\{1\}$ with Lück's observation [56, 2.5i] that the former case works for noncompact $G$. (Earlier, tomDieck had a narrower case [26] derived from [25].) 
Corollary 3.12 (tomDieck). Let $G$ be a locally compact Hausdorff group. Let $\mathcal{F}$ be a set of closed subgroups of $G$ preserved under finite intersections and under conjugacy. The coarse join $\mathbb{E}(\mathcal{F} \backslash G)=(\mathcal{F} \backslash G)^{\circ \aleph_{0}}$ is a final object in the full subcategory of numerable G-spaces. (Recall the isovariant $G$-map $\mathbb{E}(\mathcal{F} \backslash G) \longrightarrow E_{\mathcal{F}}^{\aleph_{0}} G$ of 2.2.)

The first case is recorded independently in [47, 4:12.4] [25, §3] after Milnor.

Corollary 3.13 (Husemöller). Let $G$ be a topological group. Then $\mathbb{E} G$ is a final object in the category of numerable G-spaces and $G$-homotopy classes of G-maps.

To state a stronger uniqueness, we require the notion of a stratified homotopy. The following definition we amplify to preorders from partial-orders [46, 2.6]; we shall need it in such generality and cannot assume closedness if $X$ is non-Hausdorff.

Definition 3.14 (Hughes). Let $\mathcal{P}$ be a set with a preorder ${ }^{7} \leqslant$. A topological space $Y$ shall be $(\mathcal{P}, \preccurlyeq)$-filtered if it is equipped with a set $\left\{Y^{a}\right\}_{a \in \mathcal{P}}$ of subspaces where $Y=\bigcup_{a \in \mathcal{P}} Y^{a}$ and $b<a$ implies $Y^{b} \subseteq Y^{a}$. A continuous function $f: Y \longrightarrow Z$ of $(\mathcal{P}, \preccurlyeq)$-filtered spaces is $(\mathcal{P}, \preccurlyeq)$-filtered if $f\left(Y^{a}\right) \subseteq Z^{a}$ for each $a \in \mathcal{P}$. In particular, a map $f: Y \longrightarrow Z$ shall be $(\mathcal{P}, \leqslant)$-stratified ${ }^{8}$ if $f\left(Y_{a}\right) \subseteq Z_{a}$ for each $a \in \mathcal{P}$, where

$$
Y_{a}:=Y^{a}-\bigcup_{b<a} Y^{b}
$$

The source $Y \times[-1,1]$ of a homotopy has stratification $(Y \times[-1,1])_{a}=Y_{a} \times[-1,1]$.

Example 3.15. Let $G$ be a topological group. Let $\mathcal{F}$ be a set of subgroups of $G$. Write $(\mathcal{F})$ for the set of $G$-conjugacy classes of elements of $\mathcal{F}$. Define a preorder $\geqslant$ on $(\mathcal{F})$ by: $(H) \geqslant(K)$ if $H$ contains a $G$-conjugate of $K$ (the reverse of $[49,3.5]$ ). Let $X$ be a $G$-space with orbit types in $\mathcal{F}$. The orbit-type filtration of $X / G$ is

$$
(X / G)^{(H)}:=X^{(H)} / G=\left\{x G \in X / G \mid \exists g \in G: H \subseteq G_{x g}\right\} .
$$

The orbit-type stratification of the orbit space $X / G$ is

$$
(X / G)_{(H)}:=X_{(H)} / G=\left\{x G \in X / G \mid \exists g \in G: H=G_{x g}\right\} .
$$

By isovariance, the map $f(2.1)$ is stratified and homotopy $\Phi / G(3.10)$ is filtered ${ }^{9}$.

Remark 3.16. In the preceding example, if $G$ is a Lie group and $\mathcal{F} \subseteq \operatorname{cpt}(G)$, then it follows from Cartan's closed-subgroup theorem that $\geqslant$ is moreover a partial-order. However, even for the solvable Baumslag-Solitar group

$$
G=B S(1,4)=\left\langle x, y \mid y x y^{-1}=x^{4}\right\rangle \cong \mathbb{Z}\left[\frac{1}{4}\right] \rtimes_{4} \mathbb{Z},
$$

which is a 0-dimensional Lie group with the discrete topology, antisymmetry of $\geqslant$ fails for $\mathcal{F}=\left\{\langle x\rangle,\left\langle x^{2}\right\rangle\right\}$. Therefore, for general $G$ and $\mathcal{F}$, our Definition 3.14 of filtered spaces is stated in terms of preorders, not the more familiar partial-orders.

\footnotetext{
${ }^{7}$ Recall a preorder is a partial-order without antisymmetry: $a \leqslant a$ holds and $a \leqslant c$ if $a \leqslant b \leqslant c$.

${ }^{8}$ If $(\mathcal{P}, \preccurlyeq)$ has upper topology [3, before II], $\preccurlyeq$ satisfies antisymmetry $(a=b$ if $a \preccurlyeq b \preccurlyeq a)$, and each set $Y^{a}$ is closed, then $\left\{Y_{a}\right\}_{a \in \mathcal{P}}$ is a $(\mathcal{P}, \preccurlyeq)$-stratification in the sense of Lurie [59, A.5.1]. If further the partition $\left\{Y_{a}\right\}_{a \in \mathcal{P}}$ is locally finite then it is a $(\mathcal{P}, \preccurlyeq)$-decomposition in the sense of Goresky-MacPherson [40,1.1]. If the partially ordered set is finite and $Y^{a}$ is closed cofibrant in $Y^{b}$ if $a<b$, then $\left\{Y^{a}\right\}_{a \in \mathcal{P}}$ is a $(\mathcal{P}, \preccurlyeq)$-filtration of $Y$ in the sense of Weinberger [81, p115].

${ }^{9}$ To see $\Phi / G: X / G \times \mathbb{I}_{2} \longrightarrow \mathscr{B}_{\mathcal{F}}^{I \sqcup J} G$ need not be stratified, take $K$ cohopfian in $X=K \backslash G$ and $\kappa=1, T_{1}=X=T_{2}$ and $\phi_{1}(K g)=K g, \phi_{2}(K g)=K a g$ with $a \notin N_{G}(K)$. Note $G_{H(K,-1)}=G_{(K, 0)}=K$ and $G_{H(K, 1)}=G_{(0, K a)}=a^{-1} K a$ but note $G_{H(K, 0)}=G_{(K, K a)}=K \cap a^{-1} K a \neq K$.
} 


\subsection{Arbitrary Lie groups $G$ and isometric $G$-actions}

At first, the filtered homotopy $\Phi / G(3.10)$ had a stratified strengthening [69, §2.7] [20, II:9.7].

Theorem 3.17 (Palais-Bredon). Let $G$ be a compact Lie group. Let $\mathcal{F} \subseteq \operatorname{cpt}(G)$ be finite with no conjugate elements. Consider Palais' join of Milnor's join (3.5):

$$
\mathbb{E}_{\mathcal{F}}^{n} G:=\underset{H \in \mathcal{F}}{\circledast}(H \backslash G)^{\circ n}
$$

for some $n \in \mathbb{N}$. Let $B$ be an $(\mathcal{F})$-filtered metrizable space of covering dimension $<n$. Suppose $f, g: B \longrightarrow \mathbb{B}_{\mathcal{F}}^{n} G:=\mathbb{E}_{\mathcal{F}}^{n} G / G$ are stratified maps. If $f^{*}\left(\mathbb{E}_{\mathcal{F}}^{n} G\right)$ and $g^{*}\left(\mathbb{E}_{\mathcal{F}}^{n} G\right)$ are $G$ homeomorphic over the identity $\mathrm{id}_{B}$, then there exists a stratified homotopy from $f$ to $g$.

We generalize $G, \mathcal{F}, n$ following their strategy, but we implement it differently.

Theorem 3.18. Let $G$ be an arbitrary Lie group. Let $\mathcal{F} \subseteq \operatorname{cpt}(G)$ with no conjugate elements. Consider our unrestricted isovariant join (3.7) of copies of Milnor's infinite join:

$$
\mathbb{E}_{\mathcal{F}} G:=\underset{H \in \mathcal{F}}{\forall} \mathbb{E}(H \backslash G)
$$

Let $B$ be an $(\mathcal{F})$-filtered metrizable space. Suppose $f, g: B \longrightarrow \mathbb{B}_{\mathcal{F}} G:=\mathbb{E}_{\mathcal{F}} G / G$ are stratified maps (3.14). If $f^{*}\left(\mathbb{E}_{\mathcal{F}} G\right)$ and $g^{*}\left(\mathbb{E}_{\mathcal{F}} G\right)$ are $G$-homeomorphic over the identity $\mathrm{id}_{B}$, then there exists a stratified homotopy from $f$ to $g$.

The proof appears after some lemmas in the spirit of the Palais-Bredon strategy.

The first lemma generalizes [20, II:9.2] without using transfinite induction. Therein, the members of $\mathcal{C}$ had dimension $\leqslant n$ and $F$ was a compact $(n-1)$-connected polytope [20, II:9.1]. Our $\mathcal{C}$ shall be the class $\mathcal{M}$ of metrizable spaces. Recall that $Z$ is an absolute extensor for $\mathcal{C}$, written $Z \in \mathrm{AE}(\mathcal{C})$, means that for any $X \in \mathcal{C}$ and closed subset $A \subset X$, any map $A \longrightarrow Z$ has an extension $X \longrightarrow Z$.

Lemma 3.19. Let $\mathcal{C}$ be a subclass of the class $\mathcal{P}$ of paracompact Hausdorff spaces, such that any closed subset of any member of $\mathcal{C}$ is a member of $\mathcal{C}$. Let $p: E \longrightarrow X$ be an F-fiber bundle with any structure group $[75,2.3]$ and $X \in \mathcal{C}$ and $F \in A E(\mathcal{C})$. For any closed subset $A$ of $X$, any section $A \longrightarrow$ E extends to a section $X \longrightarrow E$.

A structure group does not occur in [20, II:9.2] but does in his applications.

Proof. Since $X \in \mathcal{P}$, there is a $p$-trivializing locally finite open cover of $X$. The associated principal bundle has a trivializing locally finite open cover $\left\{U_{i}\right\}_{i=1}^{\infty}$ that is countable [25, Hilfsatz 2], which works also for the $F$-fiber bundle $p$. There is a closed refinement $\left\{C_{i} \subset U_{i}\right\}_{i=1}^{\infty}[31,2]$, which is locally finite and $p$-trivializing.

Write $A_{0}:=A$ and $A_{n+1}:=A_{n} \cup C_{n+1}$. Inductively assume a section $A_{n} \longrightarrow E$ exists extending $A \longrightarrow E$ for some $n>0$. Since $p^{-1}\left(C_{n+1}\right) \approx C_{n+1} \times F$, sections $C_{n+1} \longrightarrow E$ correspond bijectively to maps $C_{n+1} \longrightarrow F$. Then the section $A_{n} \cap C_{n+1} \longrightarrow E$ corresponds to a map $A_{n} \cap C_{n+1} \longrightarrow F$. Since $A_{n}$ is closed in $X$, we have $A_{n} \cap C_{n+1}$ is closed in $C_{n+1} \in \mathcal{C}$. Then there is an extension $C_{n+1} \longrightarrow F$. Equivalently, the section $A_{n} \cap C_{n+1} \longrightarrow E$ extends to a section $C_{n+1} \longrightarrow E$. By the pasting lemma, we obtain a section $A_{n+1} \longrightarrow E$. We are done by induction. 
TO Banakh proved the following observation using direct methods $[14,1.3]$. Indirectly, this already followed from Haver [42] with Dold [30, Proof 8.1].

Lemma 3.20 (Banakh). Let $W$ be a Lie group. Then Milnor's join $\mathbb{E} W \in A E(\mathcal{M})$.

For any class $\mathcal{C}$ of topological $G$-spaces, a $G$-space $Z$ is an absolute $G$-extensor for $\mathcal{C}$, written $Z \in G$ - $\mathrm{AE}(\mathcal{C})$, if for any closed $G$-subset $A \subset X \in \mathcal{C}$, any $G$-map $A \longrightarrow Z$ extends to a $G$-map $X \longrightarrow A$. Write $G$ - $\mathcal{M}$ for the class of Palais (3.8) $G$-metrizable spaces. Here, $G$-metrizable means there is a $G$-invariant metric. Furthermore, a $G$-space $Z$ is an absolute neighborhood $G$-extensor for $\mathcal{C}$, written $Z \in G$-ANE($(\mathcal{C})$, if for any closed $G$-subset $A \subset X \in \mathcal{C}$, any $G$-map $A \longrightarrow Z$ admits an extension to a $G$-map $U \longrightarrow Z$ for some $G$-neighborhood $U$ of $A$ in $X$.

Lemma 3.21. Let $K$ be a compact Lie group. For any $K$-normed linear space $V$ : a vectorspace with linear $K$-action and $K$-invariant norm, $\mathbb{E} V \in K-A N E(K-\mathcal{M})$.

A nonexample is the circle group $K=U_{1}$ and $V=C_{b}(\mathbb{C}, \mathbb{R})$ with the sup-norm, due to failure of continuity of right-action $(f \cdot g)(x):=f(x g)$ [8, Example 8:1].

Proof. The topological product $P:=(V \oplus \mathbb{R})^{\aleph_{0}}$ is the algebraic direct product of $\mathbb{R}$-vectorspaces with product topology. The topological vectorspace $P$ is metrizable and locally convex but not normable. (Also $P$ is Fréchet if and only if $V$ is Banach.) To see that $P$ is locally convex [19, §II:4.1], recall $P$ has the coarsest topology for which each $n$-th projection $P \longrightarrow V \oplus \mathbb{R}$ is continuous. The norm-topology on $V \oplus \mathbb{R}$ is the coarsest for which the norm and all of its vectorspace translations are continuous. Thus the topology on $P$ is the coarsest for which each $n$-th seminorm, given by $n$-th projection then $n$-th norm, and all coordinatewise vectorspace translations are continuous [19, §II:1.2]. So $P$ is locally convex [19, §II:4.1]. The proof that $P$ is metrizable formally generalizes that for $V=0: \mathbb{R}^{\kappa_{0}}$ [79, p547]. Lastly, $P$ is not normable because any basic open neighborhood of 0 contains a line.

Factoring through metric orbit spaces, by Tietze's extension theorem [78, Satz 3], we obtain that $\mathbb{R} \in K-\mathrm{AE}(K-\mathcal{M})$. Using the coordinate projections, observe that the $K$-action on $P$ is continuous; also $P \in K-\mathrm{AE}(K-\mathcal{M})$ if $V \in K-\mathrm{AE}(K-\mathcal{M})$. Above, we implicitly used the sup-norm on $V \oplus \mathbb{R}$, namely: $\|(v, r)\|:=\max \{\|v\|,|r|\}$.

Consider the bounded level-preserving $K$-injection from the coarse cone (3.1):

$$
\iota: C V \longrightarrow V \oplus \mathbb{R} ;[x, t] \longmapsto\left(\frac{t x}{1+\|x\|}, t\right) .
$$

The restriction $\iota \mid(C V-\{0\})$ away from the conepoint is an embedding. Note

$$
\iota^{-1}\{(v, r) \mid\|(v, r)\|<\varepsilon\}=\{[x, t] \mid t<\varepsilon\} .
$$

Thus $\iota$ is both continuous and open at the coarse conepoint. So $\iota$ is a $K$-embedding. Hence the product function $\iota^{\aleph_{0}}:(C V)^{\aleph_{0}} \longrightarrow P$ is a $K$-embedding. Since the image $\iota(C V)$ in $V \oplus \mathbb{R}$ is convex, it follows that $\iota^{\aleph_{0}}(\mathbb{E} V) \subset \iota^{\aleph_{0}}(C V)$ in $P$ is also convex. Therefore, since $\mathbb{E} V$ admits a $K$-embedding as a convex $K$-subset of a locally convex vectorspace $P$, and since $K$ is a compact Lie group, by Antonyan's partial generalization [7] of Dugundji's extension theorem, $\mathbb{E} V \in$ $K$-ANE $(K-\mathcal{M})$.

The above variations of extensor, if a member of $\mathcal{C}$ also, are spaces $Z$ which have the stated extension property specialized to when $A \longrightarrow Z$ is the identity map. They are forms of the retract notion, denoted by the letter $\mathrm{R}$ instead of $\mathrm{E}$ [45]. 
The Lie hypothesis of Lemma 3.21 is necessary; if $K$ is a non-Lie metric compact group, there are $K$-normed linear spaces not in $K$-ANE $(K-\mathcal{M})$ [8, Theorem 6]. Lemma 3.20 is a case of Banakh's lemma $[14,1.3]$ stated for all $W \in \operatorname{ANR}(\mathcal{M})$. The latter lemma shall be the $G=1$ case of the following equivariant generalization.

Theorem 3.22. Let $G$ be a Lie group. Milnor's join (3.5) defines a class-function

$$
\mathbb{E}: G-A N R(G-\mathcal{M}) \longrightarrow A R(\mathcal{M}) \cap G-\mathcal{M} \cap G-A N E(G-\mathcal{M}) ; Z \longmapsto \mathbb{E} Z:=Z^{\circ \aleph_{0}} .
$$

We mostly repeat the second half of Banakh's proof and introduce Palais actions. Also we remove his intermediate need for a convex subset and we fill in some details. I did not fully understand the first half of Banakh's proof, which involved some sort of abstract convexity structure and an appeal to the proof of Dugundji's theorem, so I replaced it with my own Lemma 3.21 which applies Antonyan's rigorous work.

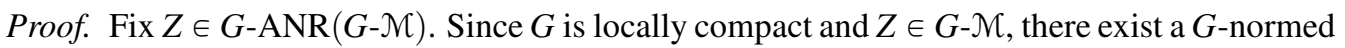
linear space $L$, a normed linear space $N$, and a closed $G$-embedding $e: Z \longrightarrow(L-0) \times N$ with open $G$-subset $L-0$ Palais [5, 3.10]. Since the $G$-action on $N$ is trivial, $(L-0) \times N$ is Palais (3.8) hence lies in $G$ - $\mathcal{M}$. Then, since $Z \in G$-ANR $(G-\mathcal{M})$, there exists a $G$-retraction $r: O \longrightarrow e(Z)$ for some $G$-neighborhood $O$ of $e(Z)$ in $(L-0) \times N \subset L \oplus N$. Consider the $G$-invariant map

$$
\eta: L \oplus N \longrightarrow[0,1] ; x \longmapsto \frac{d(x, C)}{d(x, e Z)+d(x, C)}
$$

where $C:=L \oplus N-O$ and $d(x, S):=\inf _{y \in S}\|x-y\|$. Note $\eta(C)=\{0\}$ and $\eta(e Z)=\{1\}$. So $\eta$ for $\mathcal{M}$ realizes the conclusion of Urysohn's lemma for $T_{4}$ spaces.

Using $r$ and $\eta$, next we reproduce Banakh's neighborhood retraction $R$ of $\mathbb{E}(e Z)$ in $\mathbb{E}(L \oplus N)$, and the map $R$ shall turn out to be $G$-equivariant. Define a $G$-function

$$
s: \mathbb{E}(L \oplus N) \longrightarrow[0,1] ;[x, t] \longmapsto \sum_{i=0}^{\infty} \eta\left(x_{i}\right) t_{i}
$$

To prove Banakh's assertion that $s$ is continuous, for any $i \in \mathbb{N}$ consider the function $\eta\left(x_{i}\right) t_{i}$ : $C(L \oplus N) \longrightarrow[0,1]$ defined on the coarse cone of a $G$-normed linear space. It is continuous away from the conepoint, since $\eta$ and multiplication are continuous. Given $\varepsilon>0$, taking $\delta=\varepsilon$, if $\left|t_{i}-0\right|<\delta$ then $\left|\eta\left(x_{i}\right) t_{i}-0\right| \leqslant t_{i}<\varepsilon$, so it is continuous. Thus, as the $i$-th projection is continuous, for all $n \in \mathbb{N}$, the $n$-th partial sum is too:

$$
s_{n}: C(L \oplus N)^{\aleph_{0}} \longrightarrow[0, n] ;[x, t] \longmapsto \sum_{i=0}^{n} \eta\left(x_{i}\right) t_{i} .
$$

Let $[x, t] \in \mathbb{E}(L \oplus N)$. Then $t_{i}=0$ for some $n \geqslant 0$ and all $i>n$. Let $\varepsilon>0$. Since $s_{n}$ and each $[x, t] \longmapsto t_{i}$ are continuous, then in the product topology (see 3.5), there exists an open neighborhood $U$ of $[x, t]$ in the subspace $\mathbb{E}(L \oplus N) \subset C(L \oplus N)^{\aleph_{0}}$ such that: if $\left[x^{\prime}, t^{\prime}\right] \in U$ then $\left|s_{n}[x, t]-s_{n}\left[x^{\prime}, t^{\prime}\right]\right|<\varepsilon / 2$ and $\sum_{i=0}^{n} t_{i}^{\prime}>1-\varepsilon / 2$; note

$$
\begin{aligned}
& s[x, t]-s\left[x^{\prime}, t^{\prime}\right]=s_{n}[x, t]-s\left[x^{\prime}, t^{\prime}\right] \leqslant s_{n}[x, t]-s_{n}\left[x^{\prime}, t^{\prime}\right]<\varepsilon / 2<\varepsilon \\
& s\left[x^{\prime}, t^{\prime}\right]-s[x, t]=\sum_{i>n}^{\infty} \eta\left(x_{i}^{\prime}\right) t_{i}^{\prime}+s_{n}\left[x^{\prime}, t^{\prime}\right]-s_{n}[x, t]<\sum_{i>n}^{\infty} t_{i}^{\prime}+\varepsilon / 2<\varepsilon
\end{aligned}
$$


so $\left|s[x, t]-s\left[x^{\prime}, t^{\prime}\right]\right|<\varepsilon$. Thus $s$ is continuous. Banakh's neighborhood retraction is

$$
R: s^{-1}(0,1] \longrightarrow \mathbb{E}(e Z) ;[x, t] \longmapsto\left[r\left(x_{i}\right), \frac{\eta\left(x_{i}\right) t_{i}}{s[x, t]}\right] .
$$

Let $K \in \operatorname{cpt}(G)$. By Lemma 3.21, $\mathbb{E}(L \oplus N) \in K$-ANE $(K-\mathcal{M})$ ), as $G$ hence $K$ is Lie by Cartan's closed subgroup theorem [21, 27]. Then $\mathbb{E} Z \in K-\operatorname{ANE}(K-\mathcal{M})$, as $\mathbb{E} Z$ is a neighborhood $K$-retract of $\mathbb{E}(L \oplus N)$. Thus $\mathbb{E} Z \in G$-ANE $(G-\mathcal{M})$, by Antonyan's neighborhood version [9, Thm 5] of Abels' induction theorem [1, 4.2]. Also, as $Z \in G-\mathcal{M}$, the coarse cone $C Z \in G-\mathcal{M}$ by Remark 3.2. So the induced metric $[68,20.5]$ on the countable product $(C Z)^{\aleph_{0}}$ is $G$-invariant. Hence $\mathbb{E} Z \in G$ - $\mathcal{M}$.

Finally, we establish the fact that $\mathbb{E} Z \in A E(\mathcal{M})$, independently of [14, 1.3]. Take $G=1$ in the above arguments and simplify, as follows. Take $L=0$ and the closed embedding $e: Z \longrightarrow N$ with $N$ Arens-Eells' space for $Z \in \mathcal{M}$ [13]. Obtain $R$ as above. In Lemma 3.21 for $K=1$, replace [7] with Dugundji's extension theorem [32], to find $\mathbb{E} N \in \operatorname{AE}(\mathcal{M})$. Then $\mathbb{E} Z \in \operatorname{ANE}(\mathcal{M})$, skipping [21] and [9]. So $\mathbb{E} Z \in \operatorname{AE}(\mathcal{M})$ [45, III:7.2], since $\mathbb{E} Z$ is contractible [30, 8.1].

Next, let $\mathcal{K}$ be a set of compact subgroups of a locally compact Hausdorff group $G$. Write $(G, \mathcal{K})$ - $\mathcal{M}$ for the Palais $G$-metrizable spaces with isotropy conjugate into $\mathcal{K}$.

Lemma 3.23. Fix $H \in \operatorname{lrg}(G)$ (see 2.17) for a locally compact Hausdorff group $G$. Then the Palais $G$-space $\mathbb{E}(H \backslash G)$ is a member of the class $G-A E((G,\{H\})-\mathcal{M})$.

This update of [20, II:9.3] generalizes half of a recent theorem [83, 4.3].

Corollary 3.24 (Zhang-Antonyan-Antonyan). Let $G$ be a Lie group. The trivial group 1 is a large compact subgroup of $G$, hence $\mathbb{E} G=\mathbb{E}(1 \backslash G) \in G-A E((G,\{1\})-\mathcal{M})$.

Remark 3.25. Let $G$ be a locally compact group. Updating his own earlier work, S Antonyan defines a closed subgroup $H$ as large in $G$ to be that $G / N$ is a Lie group for some normal subgroup $N \subseteq H$ of $G$ [10, 3.1]. By [10,3.2], this is equivalent to our Definition 2.17. When $G$ is separable compact Hausdorff, this equivalence is immediate from [71, Теорема 75] or [67, Theorem 6.3:1], since the kernel of the $G$-action on $G / H$ is the normal subgroup $N=$ $\bigcap_{g \in G} g \mathrm{Hg}^{-1}$.

As the large subgroup $H$ is closed in the Hausdorff group $G$, so is its normalizer $N_{G}(H)$. The closed subgroup $N_{G}(H) / N$ of the Lie group $G / N$ is Lie [21, 27]. Since $H / N$ is closed in $N_{G}(H) / N$, then $W_{G}(H):=N_{G}(H) / H$ is Lie [27, I:5.3].

Recall the $H$-skeleton (or $H$-fixed set) and $H$-stratum are the $W_{G}(H)$-spaces

$$
X^{H}:=\left\{x \in X \mid H \leqslant G_{x}\right\} \quad \text { and } \quad X_{H}:=\left\{x \in X \mid H=G_{x}\right\} .
$$

Our approach shall avoid the related and noteworthy criterion of James-Segal: for any compact Lie group $K$, a member of $K-\operatorname{ANE}(K-\mathcal{P})$ belongs to $K-\operatorname{AE}(K-\mathcal{P})$ if and only if each $H$ skeleton belongs to $\mathrm{AE}(\mathcal{P})$ for all closed $H \leqslant K[48,4.1]$.

Proof of Lemma 3.23. For easier reading, shorten $W:=W_{G}(H)$ and $E:=\mathbb{E}(H \backslash G)$. Observe that $E$ has all orbit types ${ }^{10} \sqsupseteq(H)$ and that $E^{H}=\mathbb{E}\left(H \backslash N_{G}(H)\right)=\mathbb{E} W$.

\footnotetext{
${ }^{10}$ Like Remark 3.16, there are large compact subgroups $H$ of locally compact Hausdorff groups $G$ and $g \in G$ with $g H g^{-1} \subsetneq H$, e.g. the infinite-dimensional toral group $H=\left(U_{1}\right)^{\aleph_{0}}$ in $G=H \rtimes \mathbb{Z}$.
} 
Recall from $[49,2.6]$ the space $M_{G}(X, Y):=\left\{(x, y) \in X \times Y \mid G_{x} \leqslant G_{y}\right\} / G$. Let $X$ be a $G$-metrizable space with Palais $G$-action of single orbit type $(H)$. Note

$$
M_{G}(X, E)=X_{H} \times_{N_{G}(H)} E^{H}=X_{H} \times_{W} \mathbb{E} W .
$$

The map $\pi: M_{G}(X, E) \longrightarrow X / G$ becomes $X_{H} \times_{W} \mathbb{E} W \longrightarrow X_{H} / W$, which is an $\mathbb{E} W$-fiber bundle, as $W$ is Lie (Remark 3.25), by Palais' slice theorem [70, 2.3.1].

Suppose that $A$ is a closed $G$-subset of $X$ and $f: A \longrightarrow E$ is a $G$-map. The $G$-extensions of $f$ to $X$ correspond bijectively $[49,2.6]^{11}$ to the extensions of the $\pi$-section $\Gamma f: A / G=$ $A_{H} / W \longrightarrow X_{H} \times_{W} \mathbb{E} W$ to $\pi$-sections from $X / G=X_{H} / W$. The latter exists by Lemma 3.19, since $\mathbb{E} W \in \mathrm{AE}(\mathcal{M})(3.20)$ and $\mathcal{M} \subset \mathcal{P}[77]$.

Extending the above notions, the $(H)$-skeleton and $(H)$-stratum are $G$-spaces

$$
X^{(H)}:=\left\{x \in X \mid \exists g \in G: H \leqslant G_{x g}\right\} \text { and } X_{(H)}:=\left\{x \in X \mid \exists g \in G: H=G_{x g}\right\} .
$$

Lemma 3.26. Let $H$ be a compact subgroup of a Lie group $G$. Let $X$ be a Tikhonov space with Palais $G$-action. Both $X^{(H)}$ and $X^{(H)}-X_{(H)}$ are closed subsets of $X$.

So $X_{(H)}$ is locally closed in $X$ with closure $\subseteq X^{(H)}$; see [20, p68] [27, I:6.2]. Therefore, $X$ satisfies the Frontier Condition over the poset (cpt, $\geqslant)$ [40, I:1.1].

Proof. We use the notation of Example 3.15. Let $x \in X-X^{(H)}$. Then $(H) \$\left(G_{x}\right)$. By Palais' slice theorem [70, 2.3.1], there exist a $G$-neighborhood $U$ of $x G$ in $X$ and a $G$-retraction $U \longrightarrow x G \approx G_{x} \backslash G$. If $y \in U$ then $\left(G_{y}\right) \leqslant\left(G_{x}\right)$ so $(H) 末\left(G_{y}\right)$. Thus $U \subseteq X-X^{(H)}$. Therefore $X^{(H)}$ is closed in $X$.

Let $a \in X_{(H)}$. Then $(H)=\left(G_{a}\right)$. By Palais' slice theorem, there exist a $G$-neighborhood $O$ of $a G$ in $X$ and $G$-retraction $O \longrightarrow a G \approx G / H$. Since $G$ is Lie, its closed subgroups are cohopfian, so the preorder $\leqslant$ is antisymmetric; see Footnote 10 and [27, I:3.7]. If $b \in X^{(H)} \cap O$ then $(H) \leqslant\left(G_{b}\right) \leqslant(H)$ so $(H)=\left(G_{b}\right)$. Thus $X^{(H)} \cap O \subseteq X_{(H)}$. So $X_{(H)}$ is open in $X^{(H)}$. Hence $X^{(H)}-X_{(H)}$ is closed in $X$.

Finally, we update Palais-Bredon's key cone lemma [69, §2.7] [20, II:9.4]. They only had considered compact $G$, so they equivalently used the fine cone (3.2).

Lemma 3.27. Fix a compact subgroup $H$ of an arbitrary Lie group $G$. Let $A$ be a closed $G$ subset of a Palais $G$-metrizable space $X$. Any $G$-map $\varphi: A \longrightarrow C \mathbb{E}(H \backslash G)$ with $0 \notin \varphi\left(A_{(H)}\right)$ admits a $G$-extension $\Phi: X \longrightarrow C \mathbb{E}(H \backslash G)$ such that $0 \notin \Phi\left(X_{(H)}\right)$.

Proof. Since $\varphi$ is equivariant, note $\varphi\left(A^{(H)}-A_{(H)}\right)=\{0\}$, the coarse conepoint. Write $Z:=$ $\varphi^{-1}(0)$ and $E:=\mathbb{E}(H \backslash G)$. Since $A_{(H)} \cap Z=\varnothing$, there are coordinates

$$
\varphi \mid A_{(H)}=\left(\varphi_{0}, \varphi_{1}\right): A_{(H)} \longrightarrow E \times(0,1] .
$$

Since $H \in \operatorname{lrg}(G)$ and since $A_{(H)}$ is closed in $X_{(H)} \in(G,\{H\})-\mathcal{M}$, by Lemma 3.23, $\varphi_{0}: A_{(H)} \longrightarrow$ $E$ extends to a $G$-map $\varphi_{0}^{\prime}: X_{(H)} \longrightarrow E$. Also, since $A_{(H)}$ is closed in $X_{(H)} \in \mathcal{M}$, by Tietze's extension theorem [78, Satz 3], $\varphi_{1}: A_{(H)} \longrightarrow(0,1]$ extends to a $G$-map $\varphi_{1}^{\prime}: X_{(H)} \longrightarrow(0,1]$.

\footnotetext{
${ }^{11}$ Indeed $E \in \mathcal{M} \subset T_{3.5}$, since $H \backslash G \in \mathcal{M}(2.17)$ so $C(H \backslash G), C(H \backslash G)^{\aleph_{0}} \in \mathcal{M}$ (3.2) [35, 4.2.4].
} 
Since $A_{(H)}$ and $B:=X^{(H)}-X_{(H)}$ are disjoint sets (with $B$ closed in $X$ by Lemma 3.26), similar to $(3.1)$, construct a map

$$
\eta: X^{(H)} \longrightarrow[0,1] ; x \longmapsto \frac{d(x, B)}{d\left(x, A_{(H)}\right)+d(x, B)}
$$

satisfying $\eta(B)=\{0\}$ and $\eta\left(A_{(H)}\right)=\{1\}$. Then $\varphi_{1}$ extends to $\varphi_{1}^{\prime} \eta: X^{(H)} \longrightarrow[0,1]$ which has $B$ being the preimage of 0 . So $\varphi \mid A_{(H)}$ extends to a $G$-map $\varphi^{\prime}:=\left(\varphi_{0}^{\prime}, \varphi_{1}^{\prime} \eta\right): X^{(H)} \longrightarrow C E$ with $\varphi^{\prime}(B)=\{0\}$. As $X^{(H)}$ is closed in $X$ by Lemma 3.26, by pasting lemma [68, 18.3], $\varphi$ and $\varphi^{\prime}$ unite to a $G$-map $\varphi^{\prime \prime}: A \cup X^{(H)} \longrightarrow C E$.

Again as above, the restriction of this new function has coordinates

$$
\varphi^{\prime \prime} \mid A^{\prime}=\left(\varphi_{0}^{\prime \prime}, \varphi_{1}^{\prime \prime}\right): A^{\prime}:=(A-Z) \cup X_{(H)} \longrightarrow E \times(0,1] .
$$

Since $H$ is a compact subgroup of the Lie group $G$, the orbit $H \backslash G \in G$-ANR $(G$ - $\mathcal{M})$ by Palais' slice theorem [70, 2.3.1]. Then $E \in G-\operatorname{ANE}(G-\mathcal{M})$ by Theorem 3.22. So $\varphi_{0}^{\prime \prime}: A^{\prime} \longrightarrow E$ extends to a $G$-map $\Phi_{0}^{\prime}: U \longrightarrow E$ on a $G$-neighborhood $U$ of the closed $G$-subset $A^{\prime}$ in the $G$-metrizable space $X^{\prime}:=X-(Z \cup B)$. Indeed, by Lemma 3.26, the frontier $\partial_{X}\left(X_{(H)}\right):=\overline{X_{(H)}}-X_{(H)} \subset B$ so $\partial_{X-B}\left(X_{(H)}\right)=\varnothing$. Define a $G$-map

$$
\eta^{\prime}: X^{\prime} \longrightarrow[0,1] ; x \longmapsto \frac{d\left(x, X^{\prime}-U\right)}{d\left(x, A^{\prime}\right)+d\left(x, X^{\prime}-U\right)}
$$

with $\eta^{\prime}\left(X^{\prime}-U\right)=\{0\}$ and $\eta^{\prime}\left(A^{\prime}\right)=\{1\}$. Then $\varphi_{1}^{\prime \prime}: A^{\prime} \longrightarrow(0,1]$ extends to a map $\varphi_{1}^{\prime \prime} \eta^{\prime}: X^{\prime} \longrightarrow$ $[0,1]$. So $\varphi^{\prime \prime} \mid A^{\prime}$ extends to a $G$-map $\Phi^{\prime}:=\left(\Phi_{0}^{\prime}, \varphi_{1}^{\prime \prime} \eta^{\prime}\right): X^{\prime} \longrightarrow C E$ with $\left(\Phi^{\prime}\right)^{-1}\{0\}=X^{\prime}-U$. Extend $\Phi^{\prime}$ by zero to a $G$-map $\Phi: X^{\prime} \cup Z \cup B \longrightarrow C E$.

Recall that any $G$-map $f: X \longrightarrow Y$ satisfies $G_{x} \leqslant G_{f x}$ for all $x \in X$. Furthermore, if $G_{x}=G_{f x}$ for all $x \in X$, the $G$-equivariant map $f$ is called $G$-isovariant.

Theorem 3.28. Let $G$ be a Lie group. Let $\mathcal{F} \subseteq \operatorname{cpt}(G)$ have no conjugate elements. Then $\mathbb{E}_{\mathcal{F}} G=\uplus_{H \in \mathcal{F}} \mathbb{E}(H \backslash G)$ is an isovariant absolute $G$-extensor for $(G, \mathcal{F})-\mathcal{M}$ : for any closed $G$ subset $A$ of any member $X$ of the class $(G, \mathcal{F})-\mathcal{M}$, any isovariant $G$-map $\varphi: A \longrightarrow \mathbb{E}_{\mathcal{F}} G$ extends to an isovariant $G$-map $\Phi: X \longrightarrow \mathbb{E}_{\mathcal{F}} G$.

Differently from Palais-Bredon's construction of an isovariant $\mathcal{F}$-classifying space, S Ageev asserted that $(C(\mathcal{F} \backslash G))^{\aleph_{0}}$ is an isovariant absolute $G$-extensor for $(G, \mathcal{F})-\mathcal{M}$, if $G$ is a compact Hausdorff group and $\mathcal{F} \subseteq \operatorname{lrg}(G)$ need not be finite [2, 3.2].

Proof. Write $E_{H}:=\mathbb{E}(H \backslash G)$ and denote coordinates $\varphi=\left(\varphi_{H}: A \longrightarrow C E_{H}\right)_{H \in \mathcal{F}}$. Let $H \in$ $\mathcal{F}-\{G\}$. Since $\varphi$ is isovariant, $*$ is our unrestricted isovariant join (3.7), and $\mathcal{F}$ has no conjugate elements, note for all $a \in A_{(H)}$ that

$$
(G) \neq(H)=\left(G_{a}\right)=\left(G_{\varphi a}\right)=\left(G_{\varphi_{H} a}\right) .
$$

So $0 \notin \varphi_{H}\left(A_{(H)}\right)$. By Lemma 3.27, $\varphi_{H}$ extends to a $G$-map $\Phi_{H}: X \longrightarrow C E_{H}$ with $0 \notin \Phi_{H}\left(X_{(H)}\right)$. If $G \in \mathcal{F}$ then $\varphi_{G}: A \longrightarrow C E_{G}=[0,1]$, where $0 \notin \varphi_{G}\left(A_{G}\right)$ since $\{0\}^{I} \notin \varphi\left(A_{G}\right)$, extends to a $G$-map $\Phi_{G}: X \longrightarrow[0,1]$ with $0 \notin \Phi_{G}\left(X_{G}\right)$, by [78, Satz 3] via orbit spaces to $(0,1] \in \operatorname{AE}(\mathcal{M})$ then $[0,1]$; this is if $G$ is compact and $X$ has a $G$-fixed point. So the $G$-map $\Phi:=\left(\Phi_{H}\right)_{H \in \mathcal{F}}$ : $X \longrightarrow \mathbb{E}_{\mathcal{F}} G$ is isovariant. 
Consequently, we obtain the desired corollary which is the first half of uniqueness.

Proof of Theorem 3.18. Assume a $G$-homeomorphism $\psi: f^{*}\left(\mathbb{E}_{\mathcal{F}} G\right) \longrightarrow g^{*}\left(\mathbb{E}_{\mathcal{F}} G\right)$ satisfying $\psi / G=\operatorname{id}_{B}$. Note $X:=f^{*}\left(\mathbb{E}_{\mathcal{F}} G\right) \times[0,1]$ has orbit space $X / G=B \times[0,1]$. On the closed $G$-subset $A:=f^{*}\left(\mathbb{E}_{\mathcal{F}} G\right) \times\{0,1\}$ of $X$, define the isovariant $G$-map

$$
\varphi: A \longrightarrow \mathbb{E}_{\mathcal{F}} G ;(b, e, s) \longmapsto \begin{cases}e & \text { if } s=0 \\ \psi_{1}(b, e) & \text { if } s=1\end{cases}
$$

with the pullback $f^{*}\left(\mathbb{E}_{\mathcal{F}} G\right):=\left\{(b, e) \in B \times \mathbb{E}_{\mathcal{F}} G \mid f(b)=e G\right\}$ and $\psi=\left(\operatorname{id}_{B}, \psi_{1}\right)$. Note $(\varphi / G)(b, 0)=f(b)$ and $(\varphi / G)(b, 1)=\psi_{1}(b, e) G=g(b)$. Therefore, we conclude the existence of a stratified homotopy $\Phi / G: B \times[0,1] \longrightarrow \mathbb{B}_{\mathcal{F}} G$ from $f$ to $g$ by Theorem 3.28, once we verify that $X$ is $G$-metrizable, as $X$ has isotropy in $(\mathcal{F})$.

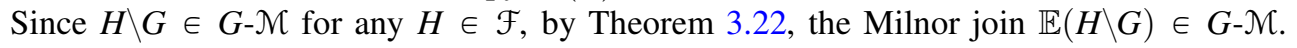
The induced metric (3.2) on its coarse cone is $G$-metrizable. The Lie group $G$ has only countably many conjugacy classes of compact subgroups, by [49, Corollary 3.9]. Then the countable product $\prod_{H \in \mathcal{F}} C \mathbb{E}(H \backslash G)$ has an induced metric [68, 20.5], whose formula is $G$-invariant. So $\mathbb{E}_{\mathcal{F}} G \in G-\mathcal{M}$. Therefore, since $B \in \mathcal{M}$, the subproduct $f^{*}\left(\mathbb{E}_{\mathcal{F}} G\right) \subset B \times \mathbb{E}_{\mathcal{F}} G \in G$ - $\mathcal{M}$, hence $X$ is also a member.

\section{The classifying property: uniqueness, II}

The following Covering Homotopy Theorem is a nontrivial result on product structures for Hausdorff $B$. In the free case, it is $[75,11.3]$ if $B$ is normal Lindelöf and locally compact, and more generally $[47,4: 9.8]$ if $B$ is paracompact. ${ }^{12}$ If $G$ is a compact Lie group, the result generalizes [69, 2.4.1] if $B$ is second-countable locally compact, and more generally [20, II:7.1] if $B$ is hereditarily paracompact.

Theorem 4.1. Let $X$ be a Tikhonov space with Palais action of a Lie group G. Suppose the orbit map is $p: X \longrightarrow B \times[0,1]$ for some hereditarily paracompact Hausdorff space B. Assume $\left(G_{x}\right)=\left(G_{y}\right)$ if $\operatorname{proj}_{B}(p x)=\operatorname{proj}_{B}(p y)$. Then $X$ is G-homeomorphic over the identity $\mathrm{id}_{B \times[0,1]}$ to the product space $p^{-1}(B \times\{0\}) \times[0,1]$.

Our ensuing proof applies and extends Palais-Bredon's argument [20, II:7.1].

Lemma 4.2. Let $(y, t) \in B \times[0,1]$. Then $p^{-1}(U \times[a, b])$ is G-homeomorphic over id to $p^{-1}(U \times$ $a) \times[a, b]$ for some neighborhoods $U$ of $y$ in $B$ and $[a, b]$ of $t$ in $[0,1]$. Furthermore, the $G$ homeomorphism restricts to id : $p^{-1}(U \times a) \longrightarrow p^{-1}(U \times a) \times a$.

We modify [20, Proof II:7.1A] to include all strata and to exclude induction.

Proof. Let $x \in p^{-1}(y, t)$. Since $X$ is Tikhonov with Palais $G$-action, by Palais' slice theorem [70, 2.3.1], there exists a $G_{x}$-slice $S$ at $x$ in $X$. The tube $S G$ is open in $X$, hence its image $p(S G)$ is open in the orbit space $X / G=B \times[0,1]$. By the tube lemma, there are neighborhoods $U$ of $y$ in $B$ and $[a, b]$ of $t$ in $[0,1]$ such that $U \times[a, b] \subseteq p(S G)$. We may assume equality by reassigning $S$ as $S \cap p^{-1}(U \times[a, b])$.

\footnotetext{
${ }^{12}$ Paracompact Hausdorff $B$ admit a product-structure theorem for microbundles [65, 3.1].
} 
Since $G_{x}$ is a compact Lie group, $S / G_{x}=S G / G=U \times[a, b]$, and $U$ is hereditarily paracompact, by [20, Theorem II:7.1], $S$ is $G_{x}$-homeomorphic over $\operatorname{id}_{U \times[a, b]}$ to the product $T \times[a, b]$ with $T:=S \cap p^{-1}(U \times a)$ and $[a, b]$ trivial $G_{x}$-action. Note

$$
p^{-1}(U \times[a, b])=S G=S \times_{G_{x}} G \approx\left(T \times_{G_{x}} G\right) \times[a, b]=p^{-1}(U \times a) \times[a, b] .
$$

Lemma 4.3. Let $y \in B$. The preimage $p^{-1}(U \times[0,1])$ is G-homeomorphic over $\mathrm{id}_{U \times[0,1]}$ to the product $p^{-1}(U \times 0) \times[0,1]$ for some neighborhood $U$ of $y$ in $B$. Furthermore, the $G$ homeomorphism restricts to id : $p^{-1}(U \times 0) \longrightarrow p^{-1}(U \times 0) \times 0$.

Our argument reexplains [20, Proof II:7.1B] but now includes all the strata.

Proof. For each $t \in[0,1]$, by Lemma 4.2, there exist a neighborhood $U_{t}$ of $y$ in $B$, a neighborhood $\left[a_{t}, b_{t}\right]$ of $t$ in $[0,1]$, and a $G$-homeomorphism $\phi_{t}$ over the identity:

$$
\phi_{t}: p^{-1}\left(U_{t} \times\left[a_{t}, b_{t}\right]\right) \longrightarrow p^{-1}\left(U_{t} \times a_{t}\right) \times\left[a_{t}, b_{t}\right] \quad \text { with } \quad \phi_{t} \mid p^{-1}\left(U_{t} \times a_{t}\right)=\text { id } .
$$

Since $[0,1]$ is compact, there is a finite subset $F \subset[0,1]$ with $(0,1)=\bigcup_{t \in F}\left(a_{t}, b_{t}\right)$. Define $U:=\bigcap_{t \in F} U_{t}$, a neighborhood of $y$ in $B$. By Lebesgue's number lemma, there is $n \in \mathbb{N}$ such that each $\left[\frac{i}{n}, \frac{i+1}{n}\right] \subseteq\left[a_{t_{i}}, b_{t_{i}}\right]$ for some $t_{i} \in F$. Thus we obtain

$$
\begin{gathered}
\varphi_{i}: p^{-1}\left(U \times\left[\frac{i}{n}, \frac{i+1}{n}\right]\right) \longrightarrow p^{-1}(U \times 0) \times\left[\frac{i}{n}, \frac{i+1}{n}\right] \\
\varphi_{i} \mid=\phi_{t_{0}} \circ \cdots \circ \phi_{t_{i-1}}: p^{-1}\left(U \times \frac{i}{n}\right) \longrightarrow p^{-1}(U \times 0) .
\end{gathered}
$$

Then $\psi:=\varphi_{0} \cup \cdots \cup \varphi_{n-1}: p^{-1}(U \times[0,1]) \longrightarrow p^{-1}(U \times 0) \times[0,1]$ as desired.

We shall avoid Bredon's transfinite induction by a Milnor-style replacement trick. Our statement is more generally in terms of predicates $\Pi$ (that is, unary relations).

Proposition 4.4. Let $(B, \mathcal{T})$ be a normal Hausdorf space. Let $\Pi \subseteq \mathcal{T}$ be preserved under all open subsets and all disjoint unions. Suppose $U \subseteq \Pi$ for a locally finite open cover $U$ of $B$. Then $\mathcal{V} \subseteq \Pi$ for a countable locally finite open cover $\mathcal{V}$ of $B$.

For local trivializations, [25, Hilfsatz 2] [47, 4:12.1] work. Originally, Milnor proved it for $(B, \mathcal{T})$ paracompact and no input $\mathcal{U}[64, \mathrm{p} 25-26][66,5.9]$.

Proof. Since $B \in T_{4}$, by Dieudonné's shrinking lemma [29, Théorème 6] and Urysohn's lemma [80, Satz 25], it follows as noted in [62, Proposition 2] that $\mathcal{U}$ admits a subordinate partition of unity $\left\{t_{U}: B \longrightarrow[0,1]\right\}_{U \in U}$ with the same index set. That is, $t_{U}$ are continuous with $\left\{t_{U}>0\right\} \subseteq U$ and $\sum_{U \in \mathcal{U}} t_{U}=1$.

We may assume $\mathcal{U}$ is infinite. For each nonempty finite $F \subset \mathcal{U}$, define the function

$$
q_{F}:=\max \left\{0, \min _{U \in F} t_{U}-\max _{U \notin F} t_{U}\right\}: B \longrightarrow[0,1] .
$$

Observe that $q_{F}$ is continuous, since the min is over the finite set $F$ and each $x \in B$ admits a neighborhood $N$ meeting only finitely many elements of $\mathcal{U}$ hence of $\mathcal{U}-F$. Then its support $V_{F}:=\left\{q_{F}>0\right\}$ is open. So $V_{F} \in \Pi$ since $V_{F} \subseteq U$ for some $U \in F$.

Let $E \neq F$ be finite subsets of $\mathcal{U}$ with $\operatorname{card} E=\operatorname{card} F$. We show $V_{E} \cap V_{F}=\varnothing$. As $E$ and $F$ are distinct of same cardinality, there are $O \in E-F$ and $P \in F-E$. If $x \in V_{E}$ 
then $t_{O}(x) \geqslant \min _{U \in E} t_{U}(x)>\max _{U \notin E} t_{U}(x) \geqslant t_{P}(x)$. Similarly, if $x \in V_{F}$ then $t_{P}(x) \geqslant \min _{U \in F} t_{U}(x)>$ $\max _{U \notin F} t_{U}(x) \geqslant t_{O}(x)$. Thus $V_{E} \cap V_{F}=\varnothing$, as $t_{O}>t_{P}>t_{O}$ on $V_{E} \cap V_{F}$. Then the union $V_{n}:=\bigcup\left\{V_{F} \mid \operatorname{card} F=n\right\}$ is disjoint. Therefore $V_{n} \in \Pi$.

Define $\mathcal{V}:=\left\{V_{n} \mid n \in \mathbb{Z}_{>0}\right\}$, a countable collection of open sets in $B$. We show $\mathcal{V}$ is a cover of $B$. Let $x \in B$. Since $\sum_{U \in \mathcal{U}} t_{U}(x)=1$, the set $S:=\left\{U \in \mathcal{U} \mid t_{U}(x)>0\right\}$ is nonempty finite. Then $q_{S}(x)=\min _{U \in S} t_{U}(x)>0$. So $x \in V_{S} \subseteq V_{\text {card } S}$. Lastly, we show $\mathcal{V}$ is locally finite. As $\mathcal{U}$ is locally finite, there is a neighborhood $N$ of $x$ in $B$ with $R:=\{U \in \mathcal{U} \mid N \cap U \neq \varnothing\}$ finite. Suppose $N \cap V_{F} \neq \varnothing$. Then $q_{F}(y)>0$ for some $y \in N$. So $t_{U}(y)>0$ hence $y \in U$ for all $U \in F$. Thus $F \subseteq R$. That is, $\left\{F \mid N \cap V_{F} \neq \varnothing\right\} \subseteq 2^{R}$, which is a finite set. Therefore $\mathcal{V}$ is locally finite.

We simplify [20, Proof II:7.1C] to include all strata and no infinite ordinals. For ease of reading, we drop the $\times 0$ for $p$-preimages that occur from Lemma 4.3. The key idea is to construct a $G$-isotopy $\delta$ on the overlap for continuous transition.

Proof of Theorem 4.1. Since $B$ is paracompact Hausdorff hence normal by [29, Théorème 1], by Lemma 4.3 and Proposition 4.4, we obtain a countable locally finite open cover $\mathcal{V}=\left\{V_{n}\right\}_{n>0}$ of $B$ and $G$-homeomorphisms $\psi_{n}: p^{-1}\left(V_{n} \times[0,1]\right) \longrightarrow p^{-1}\left(V_{n}\right) \times[0,1]$ over $\operatorname{id}_{V_{n} \times[0,1]}$ restricting to id : $p^{-1}\left(V_{n} \times 0\right) \longrightarrow p^{-1}\left(V_{n}\right) \times 0$.

Consider the open sets $U_{n}:=\bigcup_{i<n} V_{i}$ in $B$, with $U_{1}=\varnothing$. Since $\mathcal{V}$ is locally finite, it suffices to recursively define similar $G$-homeomorphisms $\phi_{n}: p^{-1}\left(U_{n} \times[0,1]\right) \longrightarrow p^{-1}\left(U_{n}\right) \times[0,1]$ such that $\phi_{n+1}\left|=\phi_{n}\right|$ over $\left(U_{n}-V_{n}\right) \times[0,1]$. Define $\phi_{1}=\mathrm{id}_{\varnothing}$.

Assume $\phi_{n}$ is defined. Shorten $U:=U_{n}$ and $V:=V_{n}$, so $U_{n+1}=U \cup V$. Write

$$
\varepsilon:=\operatorname{proj}_{X} \circ \phi_{n} \circ \psi_{n}^{-1} \mid: p^{-1}(U \cap V) \times[0,1] \longrightarrow p^{-1}(U \cap V) .
$$

As $U \cup V$ is paracompact so normal, disjoint closed sets $V-U$ and $U-V$ have disjoint $c l o s e d$ neighborhoods $C_{0}$ and $C_{1}$ in $U \cup V$. By Urysohn's lemma [80, Satz 25], there is a map $f$ : $U \cup V \longrightarrow[0,1]$ with $f\left(C_{i}\right)=\{i\}$. Define a $G$-homeomorphism

$$
\delta: p^{-1}(U \cap V) \times[0,1] \longrightarrow p^{-1}(U \cap V) \times[0,1] ;(x, t) \longmapsto(\varepsilon(x, f(p x) t), t)
$$

with inverse $\delta^{-1}(y, t)=\left(\operatorname{proj}_{X} \psi_{n} \phi_{n}^{-1}(y, f(p y) t), t\right)$. Note if $p x \in V-U$ then $\delta(x, t)=(x, t)$, and if $p x \in U-V$ then $\delta(x, t)=\phi_{n} \psi_{n}^{-1}(x, t)$. Define the $G$-bijection

$$
\phi_{n+1}:=\left\{\begin{array}{r}
\phi_{n} \text { on } U-V \\
\delta \circ \psi_{n} \text { on } U \cap V \\
\psi_{n} \text { on } V-U
\end{array}\right\}: p^{-1}\left(U_{n+1} \times[0,1]\right) \longrightarrow p^{-1}\left(U_{n+1}\right) \times[0,1] .
$$

By the pasting lemma, $\phi_{n+1}$ is continuous as $f$ is constant on each neighborhood $C_{i}$; similarly for the formula of $\phi_{n+1}^{-1}$. Then $\phi_{n+1}$ is obtained. Induction is complete.

We conclude with a summary of our uniqueness results, now inclusive of noncompact $G$.

Corollary 4.5. Let $G$ be an arbitrary Lie group. Let $\mathcal{F} \subseteq \operatorname{cpt}(G)$ with no conjugate elements. Let $B$ be an $(\mathcal{F})$-filtered metrizable space. Two maps $f, g: B \longrightarrow \mathbb{B}_{\mathcal{F}} G$ are stratified-homotopic if and only if $f^{*}\left(\mathbb{E}_{\mathcal{F}} G\right)$ and $g^{*}\left(\mathbb{E}_{\mathcal{F}} G\right)$ are $G$-homeomorphic over $\mathrm{id}_{B}$. 
Proof. The reverse direction is Theorem 3.18. For the forward direction, let $h: B \times[0,1] \longrightarrow$ $\mathbb{B}_{\mathcal{F}} G$ be a stratified homotopy from $f$ to $g$. Write $X:=h^{*}\left(\mathbb{E}_{\mathcal{F}} G\right)$, which is a $G$-metrizable Palais $G$-space as shown in Proof 3.18. Hence $X$ is Tikhonov. The metrizable space $B$ is hereditarily paracompact [77]. Write $p: X \longrightarrow B \times[0,1]$. As $h$ is stratified, $\left(G_{x}\right)=\left(G_{y}\right)$ if $\operatorname{proj}_{B}(p x)=$ $\operatorname{proj}_{B}(p y)$. By Theorem 4.1, there is a $G$-homeomorphism $X \longrightarrow f^{*}\left(\mathbb{B}_{\mathcal{F}} G\right) \times[0,1]$ over id $_{B \times[0,1]}$. It restricts to a $G$-homeomorphism $g^{*}\left(\mathbb{B}_{\mathcal{F}} G\right)=p^{-1}(B \times 1) \longrightarrow f^{*}\left(\mathbb{B}_{\mathcal{F}} G\right) \times 1$.

Remark 4.6 (Baum-Connes-Higson). For $G$ a locally compact Hausdorff group, cardinal $\kappa=$ $\aleph_{0}$, family $\mathcal{F}=\mathrm{cpt}$, and $B$ a metrizable space, a weaker variation of Corollary 4.5 is sketched in [15, Appendix 3]. Their correspondence is between ordinary homotopy classes of maps $B \longrightarrow$ $B_{\mathrm{cpt}}^{\aleph_{0}} G$ and their so-called 'homotopy' classes of Palais-proper $G$-spaces over $B$, which I instead would call concordance classes.

Here is our full classification generalizing [69, 2.6.2, 2.7.10] [20, II:9.7]. We allow noncompact $G$, infinite $\mathcal{F}$, and infinite $\operatorname{dim}(B)$; thus, we fulfill and exceed Palais' ambition [70, §4.5].

Theorem 4.7. Let $G$ be an arbitrary Lie group. Let $\mathcal{F} \subseteq \operatorname{cpt}(G)$ with no conjugate elements. Let $B$ be an $(\mathcal{F})$-filtered metrizable space. Taking pullback of $\mathbb{E}_{\mathcal{F}} G$ is a bijection from stratifiedhomotopy classes of stratified maps $B \longrightarrow \mathbb{B}_{\mathcal{F}} G$ to isomorphism classes of metrizable spaces that are equipped with Palais $G$-action, isotropy conjugate to members of $\mathcal{F}$, and orbit space $B$.

Proof. Well-definition and injectivity of this correspondence are Corollary 4.5. To show surjectivity, let $X$ be a metrizable space with Palais $G$-action, isotropy conjugate into $\mathcal{F}$, and $B=X / G$ (or a stratified homeomorphism). By Antonyan-deNeymet [11, Theorem B], $X$ admits a $G$ invariant metric. As $X \in(G, \mathcal{F})-\mathcal{M}$, by Theorem 3.28, there is an isovariant $G$-map $\Phi: X \longrightarrow$ $\mathbb{E}_{\mathcal{F}} G$. The induced map $X \longrightarrow(\Phi / G)^{*}\left(\mathbb{E}_{\mathcal{F}} G\right)$ is a $G$-homeomorphism over $\operatorname{id}_{B}[49,2.5]$.

\section{Acknowledgements}

I am grateful to Dennis Burke for discussion on orthocompactness and to Klaas Pieter Hart for helping me to locate Lindelöf's relevant work. The referee's request inspired Remark 2.7.

\section{References}

[1] Abels, H., 1978. A universal proper G-space. Math. Z. 159, 143-158. doi:10.1007/BF01214487.

[2] Ageev, S.M., 2012. Универсальные $G$-пространства Пале и изовариантные абсолютные экстензоры. Математический Сборник 203, 3-34. doi:10. 1070/SM1989v063n02ABEH003290.

[3] Aleksandrov, P., 1937. Diskrete Räume. Математический Сборник 2, 501-519.

[4] Aleksandrov, P.S., Hopf, H., 1935. Topologie. Number 45 in Grundlehren der Mathematischen Wissenschaften, Springer, Berlin.

[5] Antonyan, N., Antonyan, S., Rodríguez-Medina, L., 2009. Linearization of proper group actions. Topology Appl. 156, 1946-1956. doi:10.1016/j.topol.2009.03.016.

[6] Antonyan, N., Antonyan, S., Varela-Velasco, R., 2012. Universal $G$-spaces for proper actions of locally compact groups. Topology Appl. 159, 1159-1168. doi:10.1016/j.topol.2011.11.036.

[7] Antonyan, S., 1985. Эквивариантное обобщение теоремы Дугунджи. Математические Заметки 38, 608-616. doi:10.1007/BF01158413.

[8] Antonyan, S., 1987. Equivariant embeddings into G-ARs. Glas. Mat. Ser. III 22, 503-533.

[9] Antonyan, S., 1999. Extensorial properties of orbit spaces of proper group actions. Topology Appl. 98, 35-46. doi:10.1016/S0166-8641(99)00039-5.

[10] Antonyan, S., 2005. Orbit spaces and unions of equivariant absolute neighborhood extensors. Topology Appl. 146/147, 289-315. doi:10.1016/j.topol.2003.05.004.

[11] Antonyan, S., de Neymet, S., 2003. Invariant pseudometrics on Palais proper $G$-spaces. Acta Math. Hungar. 98, 59-69. doi:10.1023/A: 1022853304454. 
[12] Arens, R., 1946. Topologies for homeomorphism groups. Amer. J. Math. 68, 593-610. doi:10. $2307 / 2371787$.

[13] Arens, R., Eells Jr, J., 1956. On embedding uniform and topological spaces. Pacific J. Math. 6, $397-403$.

[14] Banakh, T.O., 1992. О топологии конструкции Милнора универсального $G$-расслоения. Сибирский Математический ЖКурнал 33, 16-25. doi:10.1007/BF00972931.

[15] Baum, P., Connes, A., Higson, N., 1994. Classifying space for proper actions and $K$-theory of group $C^{*}$-algebras, in: $C^{*}$-algebras: 1943-1993 (San Antonio, TX, 1993). Amer. Math. Soc., Providence, RI. volume 167 of Contemp. Math., pp. 240-291. doi:10.1090/conm/167/1292018.

[16] Biller, H., 2004. Characterizations of proper actions. Math. Proc. Cambridge Philos. Soc. 136, 429-439. doi:10.1017/S0305004103007217.

[17] Björner, A., 1984. Posets, regular CW complexes and Bruhat order. European J. Combin. 5, 7-16. doi:10.1016/S0195-6698(84)80012-8.

[18] Bourbaki, N., 1961. Structures topologiques. Number I in Éléments de mathématique: Topologie générale. third (recast) ed., Actualités Sci. Indust. \#1142, Paris.

[19] Bourbaki, N., 1981. Ensembles convexes et espaces localement convexes. Number II in Éléments de mathématique: Espaces vectoriels topologiques. second (recast) ed., Masson, Paris.

[20] Bredon, G.E., 1972. Introduction to Compact Transformation Groups. Number 46 in Pure and Applied Mathematics, Academic Press, New York and London.

[21] Cartan, E., 1930. La théorie des groupes finis et continus et l'Analysis situs. Number 42 in Mémorial des Sciences Mathématiques, Gauthier-Villars, Paris.

[22] Čech, E., 1937. On bicompact spaces. Ann. of Math. (2) 38, 823-844. doi:10. 2307/1968839

[23] Cianci, N., Ottina, M., . Fiber bundles over Alexandroff spaces. https://arxiv.org/abs/1907.03614v2.

[24] Crowell, R.H., 1963. Invertible isotopies. Proc. Amer. Math. Soc. 14, 658-664. doi:10.2307/2034296.

[25] tom Dieck, T., 1966. Klassifikation numerierbarer Bündel. Arch. Math. (Basel) 17, $395-399$. doi:10.1007/BF01899618.

[26] tom Dieck, T., 1972. Orbittypen und äquivariante Homologie, I. Arch. Math. (Basel) 23, $307-317$. doi:10.1007/BF01304886.

[27] tom Dieck, T., 1987. Transformation Groups. Number 8 in De Gruyter Studies in Mathematics, Walter de Gruyter, Berlin. doi:10.1515/9783110858372.312.

[28] Dietze, A., Schaps, M., 1974. Determining subgroups of a given finite index in a finitely presented group. Canadian J. Math. 26, 769-782. doi:10.4153/CJM-1974-072-0.

[29] Dieudonné, J., 1944. Une généralisation des espaces compacts. J. Math. Pures Appl. (9) 23, 65-76.

[30] Dold, A., 1963. Partitions of unity in the theory of fibrations. Ann. of Math. (2) 78, 223-255. doi:10.2307/1970341.

[31] Dowker, C., 1951. On countably paracompact spaces. Canadian J. Math. 3, $219-224$. doi:10.4153/c jm-1951-026-2.

[32] Dugundji, J., 1951. An extension of Tietze's theorem. Pacific J. Math. 1, 353-367.

[33] Ehresmann, C., Feldbau, J., 1941. Sur les propriétés d'homotopie des espaces fibrés. C. R. Acad. Sci. Paris 212, 945-948.

[34] Elmendorf, A.D., 1983. Systems of fixed point sets. Trans. Amer. Math. Soc. 277, 275-284 doi:10.2307/1999356.

[35] Engelking, R., 1989. General Topology. Number 6 in Sigma Series in Pure Mathematics. second (expanded) ed., Heldermann Verlag, Berlin. Translated from the Polish.

[36] Fox, R., 1945. On topologies for function spaces. Bull. Amer. Math. Soc. 51, 429-432. doi:10.1090/S0002-9904-1945-08370-0

[37] Fréchet, M., 1918. Sur la notion de voisinage dans les ensembles abstraits. Bull. Sci. Math. (2) 42, 138-156.

[38] Fritsch, R., Golasiński, M., 2004. Topological, simplicial and categorical joins. Arch. Math. (Basel) 82, 468-480. doi:10.1007/s00013-003-0431-y

[39] Gelfand, I.M., Fuks, D.B., 1968. О классифицирующих пространствах для главных расслоений с хаусдорфовыми базами. Доклады Академии Наук СССР 181, 515-518.

[40] Goresky, M., MacPherson, R., 1988. Stratified Morse theory. Number 14 in Ergebnisse der Mathematik und ihrer Grenzgebiete (3), Springer-Verlag, Berlin. doi:10.1007/978-3-642-71714-7.

[41] Guillou, B.J., May, J.P., Merling, M., 2017. Categorical models for equivariant classifying spaces. Algebr. Geom. Topol. 17, 2565-2602. doi:10.2140/agt.2017.17.2565

[42] Haver, W.E., 1973. Locally contractible spaces that are absolute neighborhood retracts. Proc. Amer. Math. Soc. 40, 280-284. doi:10 . 2307/2038681.

[43] Hofmann, K.H., Morris, S.A., 2006. The Structure of Compact Groups. Number 25 in Studies in Mathematics. second (augmented) ed., Walter de Gruyter, Berlin. doi:10.1515/9783110199772.

[44] Holm, P., 1967. The microbundle representation theorem. Acta Math. 117, 191-213. doi:10.1007/BF02395045.

[45] Hu, S.T., 1965. Theory of Retracts. Wayne State University Press, Detroit. 
[46] Hughes, C.B., 1999. Stratified path spaces and fibrations. Proc. Roy. Soc. Edinburgh Sect. A 129, $351-384$. doi:10.1017/S0308210500021405.

[47] Husemöller, D., 1966. Fibre Bundles. McGraw-Hill Book Co, NewYork-London-Sydney.

[48] James, I., Segal, G., 1980. On equivariant homotopy theory, in: Topology Symposium, Siegen 1979, Springer. pp. 316-330.

[49] Khan, Q., . Countable approximation of topological $G$-manifolds, III: arbitrary Lie groups $G$. https://arxiv.org/abs/1905.09977; submitted for publication.

[50] Kolmogorov, A.N., 1934. Zur Normierbarkeit eines allgemeinen topologischen linearen Raumes. Stud. Math. 5, 29-33.

[51] Lashof, R., 1982. Equivariant bundles. Illinois J. Math. 26, 257-271.

[52] Lashof, R., May, J.P., 1986. Generalized equivariant bundles. Bull. Soc. Math. Belg. A38, 265-271.

[53] Lashof, R., Rothenberg, M., 1978. G-smoothing theory, in: Algebraic and Geometric Topology, American Mathematical Society, Providence. pp. 211-266.

[54] Lefschetz, S., 1942. Topics in Topology. Number 10 in Annals of Mathematics Studies, Princeton University Press, Princeton.

[55] Lindelöf, E., 1904. Sur quelques points de la théorie des ensembles. C. R. Acad. Sci. Paris 137, 697-700.

[56] Lück, W., 2005. Survey on classifying spaces for families of subgroups, in: Infinite groups: geometric, combinatorial and dynamical aspects. Birkhäuser, Basel. volume 248 of Progr. Math., pp. $269-322$. doi:10.1007/3-7643-7447-0_7.

[57] Lück, W., Uribe, B., 2014. Equivariant principal bundles and their classifying spaces. Algebr. Geom. Topol. 14, 1925-1995. doi:10.2140/agt.2014.14.1925.

[58] Lundell, A.T., Weingram, S., 1969. The Topology of CW Complexes. The University Series in Higher Mathematics, Van Nostrand Reinhold Co., New York. doi:10.1007/978-1-4684-6254-8.

[59] Lurie, J., . Higher Algebra. https://www.math.ias.edu/ lurie/papers/HA .pdf

[60] MacLane, S., 1971. Categories for the Working Mathematician. Number 5 in Graduate Texts in Mathematics, Springer-Verlag, New York-Berlin

[61] McCord, M.C., 1966. Singular homology groups and homotopy groups of finite topological spaces. Duke Math. J. $33,465-474$.

[62] Michael, E., 1953. A note on paracompact spaces. Proc. Amer. Math. Soc. 4, 831-838. doi:10. 2307/2032419.

[63] Milnor, J.W., 1956. Construction of universal bundles, II. Ann. of Math. (2) 63, 430-436. doi:10. $2307 / 1970012$.

[64] Milnor, J.W., 1957. Lectures on Characteristic Classes. Princeton University. Notes by James Stasheff.

[65] Milnor, J.W., 1964. Microbundles. Topology 3, 53-80. doi:10.1016/0040-9383(64)90005-9.

[66] Milnor, J.W., Stasheff, J.D., 1974. Characteristic Classes. Princeton University Press, Princeton, N. J.; University of Tokyo Press, Tokyo. Annals of Mathematics Studies, No. 76

[67] Montgomery, D., Zippin, L., 1955. Topological Transformation Groups. Interscience Publishers, New YorkLondon.

[68] Munkres, J.R., 2000. Topology. Prentice Hall, Upper Saddle River.

[69] Palais, R.S., 1960. The Classification of $G$-spaces. Number 36 in Memoirs of the AMS, American Mathematical Society, Providence.

[70] Palais, R.S., 1961. On the existence of slices for actions of non-compact Lie groups. Ann. of Math. (2) 73, 295-323. doi:10.2307/1970335.

[71] Pontryagin, L.S., 1954. Непрерывные Группы. Second (expanded) ed., Государственное Издательство Технико-Теоретической Литературы, Moscow.

[72] Segal, G., 1975. Классифицирующее пространство топологической группы в смысле ГельфандаФукса. Функциольный Анализ и его Приложения 9, 48-50. doi:10.1007/BF01075450.

[73] Sierpiński, W., 1952. General Topology. Number 7 in Mathematical Expositions. second (recast) ed., University of Toronto Press, Toronto. Translated from the Polish.

[74] Steen, L.A., Seebach Jr, J.A., 1978. Counterexamples in Topology. Second (corrected) ed., Springer Verlag, New York and Heidelberg.

[75] Steenrod, N.E., 1951. The Topology of Fibre Bundles. Number 14 in Princeton Mathematical Series, Princeton University Press, Princeton.

[76] Steenrod, N.E., 1967. A convenient category of topological spaces. Michigan Math. J. 14, 133-152.

[77] Stone, A.H., 1948. Paracompactness and product spaces. Bull. Amer. Math. Soc. 54, 977-982. doi:10.1090/S0002-9904-1948-09118-2.

[78] Tietze, H., 1915. Über Funktionen, die auf einer abgeschlossenen Menge stetig sind. J. Reine Angew. Math. 145, 9-14. doi:10.1515/crll.1915.145.9.

[79] Tikhonov, A.N., 1930. Über die topologische Erweiterung von Räumen. Math. Ann. 102, 544-561. doi:10.1007/BF01782364.

[80] Urysohn, P., 1925. Über die Mächtigkeit der zusammenhängenden Mengen. Math. Ann. 94, $262-295$. 
doi:10.1007/BF01208659.

[81] Weinberger, S., 1994. The Topological Classification of Stratified Spaces. Chicago Lectures in Mathematics, University of Chicago Press, Chicago.

[82] Whitehead, J.H.C., 1939. Simplicial spaces, nuclei and m-groups. Proc. London Math. Soc. (2) 45, $243-327$. doi:10.1112/plms/s2-45.1.243.

[83] Zhang, L.L., Antonyan, S., Antonyan, N., 2019. Universal $G$-spaces for proper free actions. Topology Appl. 264, 336-351. doi:10.1016/j.topol.2019.06.034. 